\title{
Characterizing envelopes of moving rotational cones and applications in CNC machining
}

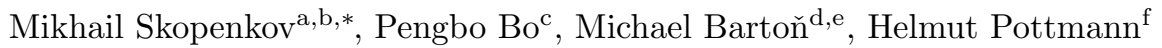 \\ ${ }^{a}$ National Research University Higher School of Economics, Faculty of Mathematics, Usacheva 6, Moscow 119048, Russia \\ ${ }^{b}$ Institute for Information Transmission Problems of the Russian Academy of Sciences, Bolshoy Karetny per. 19, build.1, Moscow 127051, Russia \\ ${ }^{c}$ School of Computer Science and Technology, Harbin Institute of Technology, West Wenhua Str. 2, 264209 Weihai, China \\ ${ }^{d}$ BCAM - Basque Center for Applied Mathematics, Alameda de Mazarredo 14, 48009 Bilbao, Basque Country, Spain \\ ${ }^{e}$ Ikerbasque - Basque Foundation for Sciences, Maria Diaz de Haro 3, 48013 Bilbao, Basque Country, Spain \\ ${ }^{f}$ King Abdullah University of Science and Technology, P.O. Box 2187, 4700 Thuwal, 23955-6900, Kingdom of Saudi Arabia
}

\begin{abstract}
Motivated by applications in CNC machining, we provide a characterization of surfaces which are enveloped by a oneparametric family of congruent rotational cones. As limit cases, we also address ruled surfaces and their offsets. The characterizations are higher order nonlinear PDEs generalizing the ones by Gauss and Monge for developable surfaces and ruled surfaces, respectively. The derivation includes results on local approximations of a surface by cones of revolution, which are expressed by contact order in the space of planes. To this purpose, the isotropic model of Laguerre geometry is used as there rotational cones correspond to curves (isotropic circles) and higher order contact is computed with respect to the image of the input surface in the isotropic model. Therefore, one studies curve-surface contact that is conceptually simpler than the surface-surface case. We show that, in a generic case, there exist at most six positions of a fixed rotational cone that have third order contact with the input surface. These results are themselves of interest in geometric computing, for example in cutter selection and positioning for flank CNC machining.
\end{abstract}

Keywords: envelope of cones, Laguerre geometry, ruled surface, higher-order contact, flank CNC machining

\section{Introduction}

Various manufacturing technologies, such as hot wire cutting, electrical discharge machining or computer numerically controlled (CNC) machining are based on a moving tool, the active part of which can be a curve or a surface. They generate surfaces which are swept by a simple curve, e.g. a straight line segment or a circular arc, or are enveloped by a simple surface. The latter case mostly refers to CNC machining where the moving tool is part of a rotational surface (sphere, rotational cylinder, rotational cone, torus). In order to produce a given shape with such a manufacturing process, one has to approximate the target shape by surfaces which are generated by a moving tool of the available type. Depending on the application, such an approximation has to be highly accurate and, for example in the case of CNC machining may have to meet a numerical tolerance of a few micrometers for objects of the size of tens of centimeters. Such high precision pushes demands on the path-planning algorithms which greatly benefit from a higher order analysis of the contact between the reference surface and the surface generated by the moving tool.

A moving tool, conceptualized as a truncated cone, in the context of 5-axis flank CNC machining is shown in Fig. 1. Its motion is visualized by the motion of its axis (tracing a ruled surface). Ideally, the tool is supposed to touch the surface not only at a single point, but along a whole curve, known as characteristic. The characteristic is the intersection of the current position of the cone with the position at an "infinitesimally close" moment, therefore is an algebraic curve of degree four. For some special instantaneous motions, e.g. translation, this characteristic can degenerate to a pair of straight lines passing through the vertex of the cone, however, for a generic screw motion, the characteristic is a spatial curve lying on the cone. Therefore solving the flank-milling problem by approximating the given surface by developable patches, as widely done in engineering literature, is not a correct approach as it restricts the space of solutions. In this work we look for good initial positions of cones that admit higher order contact with the surface.

With the flank CNC machining application in mind, we present such an analysis for envelopes of rotational cones. We emphasize here that we strictly focus on cones of revolution (aka rotational cones). A rotational cone is formed by all lines passing through a fixed point (vertex) and a fixed circle such that the orthogonal projection of the vertex to the plane of the circle is its center (but the vertex does not coincide with the center). In order to obtain contact of order $n$ between an envelope of a moving rotational cone and a design surface $\Phi$, it is not necessary that each position of the cone has contact of order $n$ with $\Phi$, when viewing the surfaces as point sets. This is obvious anyway, since 2 nd order contact between a cone and a surface

\footnotetext{
${ }^{*}$ Corresponding author

Email addresses: mikhail.skopenkov@gmail.com (Mikhail Skopenkov), pbbo@hit.edu.cn (Pengbo Bo), mbarton@bcamath.org (Michael Bartoň), helmut.pottmann@kaust.edu.sa (Helmut Pottmann)
} 

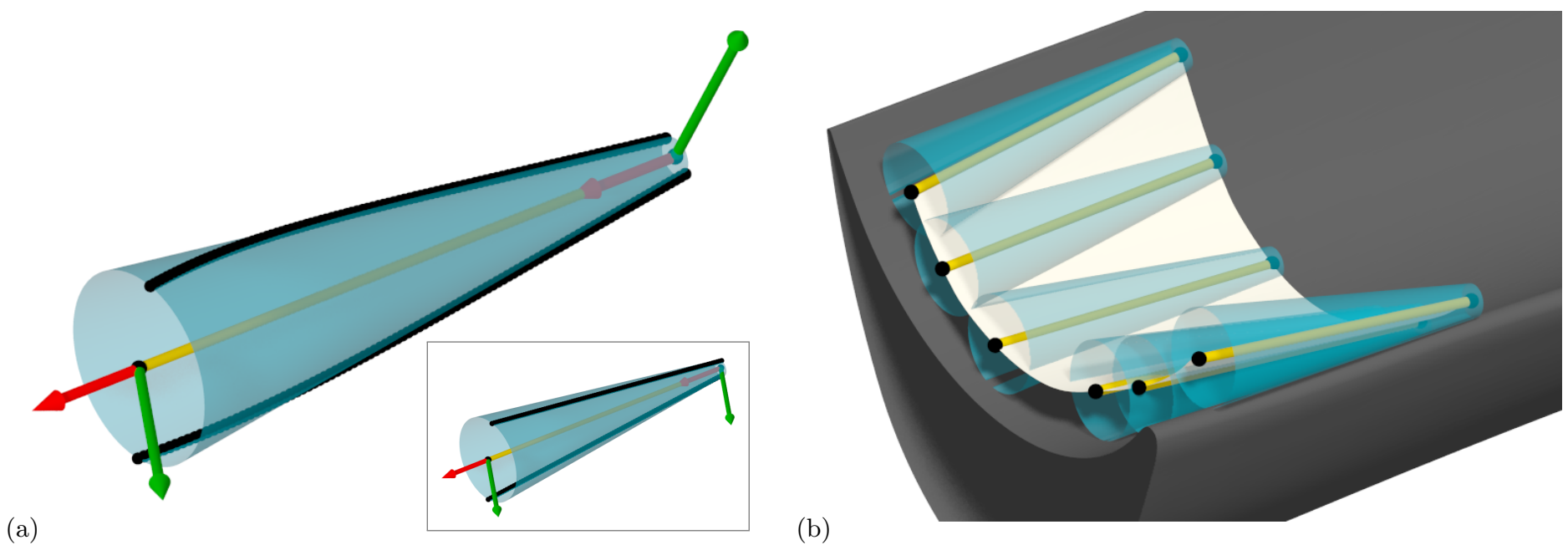

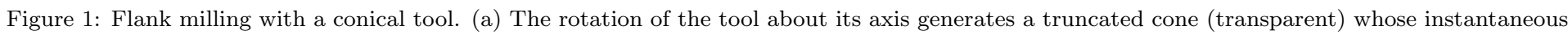

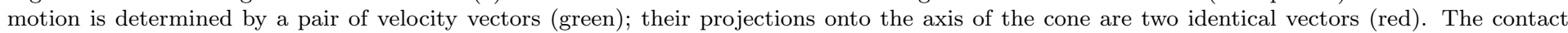

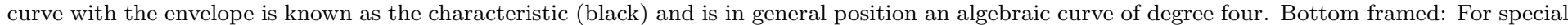

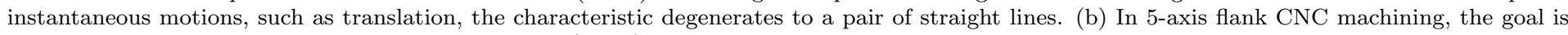

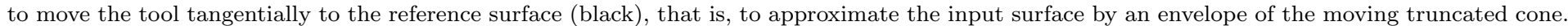

$\Phi$ would already imply vanishing Gaussian curvature of $\Phi$, i.e., a developable surface $\Phi$. One needs contact of order $n$ between the cone and the surface, viewed in the space of planes. It is related to the fact that a cone possesses just a one-parameter family of tangent planes. This indicates the advantage of using a geometry, in which the (oriented) planes in Euclidean space are the basic elements. Therefore, we use Laguerre geometry and work in a point model of the set of oriented planes, known as the isotropic model of Laguerre geometry. There, a cone appears as a curve (an isotropic circle) and not as a surface. That is, the analysis of cone-surface contact is transferred to the study of a curve-surface contact, which is conceptually simpler.

When we speak of higher order contact between a surface generated by a conical milling tool and a reference surface, it is important to note the following: Second order contact, also referred to as osculation, means that the surfaces locally penetrate tangentially. Thus, this case is not directly suitable for CNC machining, but may still be useful for initial estimates of good tool positions. However, third order contact, so-called hyperosculation, is locally penetration-free in the very neighborhood of the contact point and therefore very well-suited for CNC machining, in particular for initialization of optimization algorithms which aim at high-precision machining.

\section{Contributions and overview}

Our main contribution is a careful analysis of plane-based higher order contact between cones of revolution and a given reference surface. This leads to a nonlinear PDE which characterizes exact envelopes of congruent rotational cones (see Theorem 13). From a practical perspective, this means that we can detect the (rare) cases in which a surface can be milled exactly in a single path by flank milling with an appropriate conical tool, provided that this tool motion is collision free and accessible. Probably more importantly, a computational approach to locally well fitting tool positions is very helpful for the initialization of numerical optimization algorithms for high-precision tool motion planning. On our way towards the characterization of envelopes of moving rotational cones, we discuss other special types of surfaces as well.

The paper is structured as follows: We discuss relevant previous work in Section 2. Section 3 derives a PDE that characterizes the graph of a bivariate function as a ruled surface (Theorem 1). To extend to envelopes of cones, in Section 4 we introduce the isotropic model of Laguerre geometry and discuss the contact order between a developable surface and a doubly curved surface, expressed in the space of planes. Section 5 characterizes envelopes of congruent rotational cones in the isotropic model and formulates conditions on second order and third order plane-based contact. This is the basis for a PDE characterization of envelopes of congruent rotational cones (Section 6, Theorem 13). In Section 7 we address the limit case of envelopes of congruent rotational cylinders (Corollary 21). Section 8 shows examples of hyperosculating cone positions and its application to flank CNC machining. Finally, Section 9 concludes the paper and indicates directions for future research. In Appendix A and Appendix B we prove Theorems 1 and 10 respectively (the latter implying Theorem 13) and in the process give an "algorithm" to reconstruct rulings and special conics on a given surface (and hence cones enveloping a surface).

\section{Previous work}

\section{Geometry}

Higher order contact between curves and/or surfaces has been well-studied in the past, see e.g. [11, 29, 49]. It appears, for example, in surface-surface intersection: Using marching methods is straightforward for transversal intersections, however, when the surfaces in question have higher order contact, the computation of the intersection curve is quite complex [49]. Higher 
order contact between a circle and a surface in Euclidean 3-space is studied in [29], in particular the existence of circles with 5 -th order contact at the umbilical points of a surface.

Another class of relevant research deals with the approximation of general free-form (NURBS) surfaces by ruled surfaces [20, 44], or even developable surfaces [34, 35, 39, 41, 42]. For simple geometries, the process of approximation can be even interactive, while the design of very complex shapes requires many rounds of optimization and is still beyond real-time performance [42].

With the blossom of modern free-form architecture, another type of research appeared recently. A curved geometry on a large scale requires fine approximation in order to, for example, create panels, molds for their production and support structures. This requires segmentation of the whole complex free-form surface into manufacturable patches, while minimizing the cost of the whole manufacturing process [14]. To this end, another promising direction is to use to simple, ideally congruent, curved geometric entities such as circular arcs $[1,6]$ in a repetitive manner.

\section{CNC machining}

The problem of approximating a general free-form surface by an envelope of a moving simple object (e.g. a quadric) has been inspired by applications in 5-axis CNC machining. We refer to the very final stage of 5-axis CNC machining, known as flank machining, where the tool, typically a cone or a cylinder, moves tangentially along the to-be-manufactured surface, having a contact with the surface - theoretically - along a whole curve, see Fig. 1.

In the case of 5-axis flank milling with cylindrical tools, the tool path-finding problem can be alternatively formulated as approximating the offset surface of the input surface (offset by the radius of the tool) by a set of ruled surfaces. Therefore a lot of literature is devoted to this equivalent formulation, see e.g. [10, 18, 24, 27, 36, 40, 45, 48] and the fact that a free-form surface can be approximated by ruled surfaces arbitrarily well [15]. However, this approximation of a general, doubly-curved surface by ruled surfaces within fine tolerances typically requires an excessive number of patches [15]. On the other hand, negatively curved surfaces can be approximated even by a reasonably small number of smoothly joining ruled surface strips [17].

In the case of approximation with conical tools, the literature is a lot more sparse. One can machine a ruled surface perfectly with a cylindrical or conical tool only if the tangent plane along the ruling is constant, i.e., the surface is developable. For a general (non-developable) ruled surface, an approximation approach is necessary [24]. For general free-form surfaces, an alternative approach is to use an approximation of the surface's distance function and look for directions in which its Hessian vanishes [5]. Along these 3D directions, the distance from the reference surface changes linearly and therefore provides good initial candidates for the milling axis positions.

Another important issue is the accessibility of the surface by a machining tool. A conservative estimate is proposed in the context of 5-axis ball-end milling [16]. The admissible directions of the tool are encoded using normal bounding cones which enables to quickly find whole volumes in the configuration space that correspond to possible tool paths. As a result, there is no need to compute accessibility for individual cutter contact points which brings significant computational savings.

Real-life manufacturing of free-form surfaces using conical tools is conducted in [8]. Using the initialization strategy for flank milling with conical tools introduced in [5], one quickly finds initial motions (ruled surfaces) of the milling axis and reveals the parts of free-form surfaces that can be efficiently approximated by conical envelopes within very fine machining tolerances. Consequently, high accuracy leads to a reduced machining time as only few sweeps are needed to cover large portions of the surface [8].

Another strong stream of research deals with curved tools and especially barrels [25, 26, 43]. Barrel tools are shown to fit well free-form surfaces, especially in concave regions where the principal curvatures of the tool match their counterparts of the surface. The most recent research focuses on custom-shaped tools. That is, not only the 3D motion of the tool, but also the shape itself are the unknowns in path-planning $[19,51,52,53,54,55]$. Typically, the initial milling trajectory is a part of the input or is indicated by the user. Recent research focuses on automatic path initialization for 5-axis flank milling $[4,5]$. For a specific shape of the milling tool (conical or doubly curved), an automatic initialization of the motion of the tool can be achieved by integrating the admissible multi-valued vector field that corresponds to directions in which the point-surface distance changes according to the prescribed shape of the milling tool (prescribed by a meridian curve) [4].

On the conceptual level, our research in this paper is closely related to $[46,47,50]$, which concerns research on 5 -axis flatend milling with cylindrical tools, where the bottom circle is posed in third order contact (hyperosculation) with the reference surface. In this work, however, we have to deal with higher order contact in the space of planes, i.e., we look for hyperosculation between a special conic and a curved surface in the isotropic model of Laguerre geometry, and not in Euclidean space.

\section{Ruled surfaces}

Ruled surfaces are traced by a line moving in space. They appear as limits of surfaces enveloped by a one-parametric family of congruent rotational cones when the opening angle tends to zero whereas the vertices stay fixed. We first treat this well-known class of surfaces and in this way introduce to our approach at hand of a well-known case. A particular case of ruled surfaces are developable ones. The latter are enveloped by a one-parametric family of planes. For a developable surface, a tangent plane touches the surface along a straight line segment (ruling); see Fig. 2(a). Not all ruled surfaces are developable: at a generic, so-called non-torsal ruling of a ruled surface, the tangent plane is not constant (Fig. 2(b); for a detailed discussion, see [34]). 


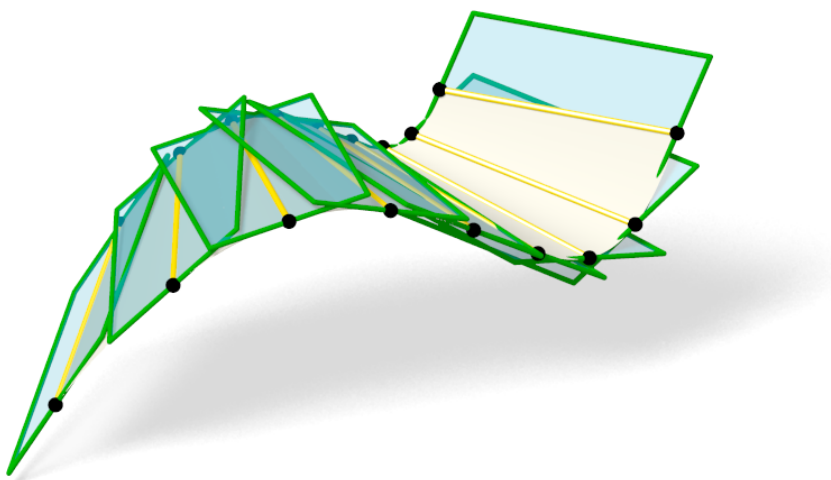

(a)

Figure 2: (a) A developable ruled surface is an envelope of a one-parameter family of planes. Every plane touches the surface along the whole ruling. In contrast, (b) tangent planes vary along a generic ruling of a non-developable ruled surface.

We derive a PDE characterizing ruled surfaces. It is by far less known than the one for developable surfaces. The classical origin is found in affine differential geometry (see Blaschke [2], cf. [28]), where ruled surfaces are characterized by the vanishing of a 3rd order differential invariant, called Pick's invariant. To our knowledge, the resulting PDE was first written explicitly by R. Bryant recently [7]. All that is equivalent to the result (Theorem 1) given below. Our approach is elementary and does not require knowledge in affine differential geometry.

Take the surface to be the graph of a $C^{3}$ function $f(x, y)$. Assume that the segment $(x+u t, y+v t, z+w t)$, where $t$ runs through $(-\varepsilon, \varepsilon)$ and $u, v, w \in \mathbb{R}$ are fixed, is contained in the graph. Then $z+w t=f(x+u t, y+v t)$ identically. Differentiating 3 times with respect to $t$ consecutively, we get

$$
\left\{\begin{array}{l}
f_{x x} u^{2}+2 f_{x y} u v+f_{y y} v^{2}=0 \\
f_{x x x} u^{3}+3 f_{x x y} u^{2} v+3 f_{x y y} u v^{2}+f_{y y y} v^{3}=0 .
\end{array}\right.
$$

The solvability of the system (1) is analysed directly. The two equations (1) have a common solution (u,v), if and only if the resultant of the left-hand-side polynomials vanishes:

$$
\begin{aligned}
f_{y y}{ }^{3} f_{x x x}{ }^{2}+6 f_{y y} f_{x x x} f_{y y y} f_{x y} f_{x x}-6 f_{y y}{ }^{2} f_{x x x} f_{x y y} f_{x x}-6 f_{y y y} f_{x y} f_{x x}{ }^{2} f_{x y y} \\
+9 f_{y y} f_{x y y}{ }^{2} f_{x x}{ }^{2}-6 f_{x y} f_{y y}{ }^{2} f_{x x y} f_{x x x}+12 f_{x y}{ }^{2} f_{x x y} f_{y y y} f_{x x}-18 f_{x y} f_{y y} f_{x x y} f_{x y y} f_{x x} \\
+12 f_{y y} f_{x y y} f_{x y}{ }^{2} f_{x x x}-8 f_{y y y} f_{x y}{ }^{3} f_{x x x}+9 f_{x x} f_{y y}{ }^{2} f_{x x y}{ }^{2}-6 f_{y y} f_{x x y} f_{y y y} f_{x x}{ }^{2}+f_{y y y}{ }^{2} f_{x x}{ }^{3}=0 .
\end{aligned}
$$

The first equation of (1) has a real solution (and moreover all solutions are proportional to real ones), if and only if

$$
f_{x x} f_{y y}-f_{x y}^{2} \leq 0
$$

i.e., the Gaussian curvature $K$ of the surface is non-positive. By the property of the resultant, (1) has a real solution ( $u, v)$, if and only if we have (2) and (3).

Geometrically, the first equation of (1) expresses that the segment is an asymptotic direction (direction of vanishing normal curvature; see [12]). If both equations in (1) are satisfied, the line in direction $(u, v, w)$ (with $\left.w=f_{x} u+f_{y} v\right)$ has $3 r d$ order contact with the surface (cf. Definition 5 below).

Conversely, if (2)-(3) hold, then the surface is ruled. In Appendix A we present an "algorithm" to reconstruct a ruling through a generic point on the surface, and prove the following theorem.

Theorem 1 (characterization of ruled surfaces). For a $C^{3}$ function $f: D \rightarrow \mathbb{R}$ defined in an open disk $D \subset \mathbb{R}^{2}$ the following 3 conditions are equivalent:

1. Through a generic point of the graph of $f$ there passes a line segment completely contained in the graph.

2. For each $(x, y) \in D$, the two equations (1) have a common nonzero real solution $(u, v)$.

3. For each $(x, y) \in D$ we have (2) and (3) (the latter meaning nonpositive Gaussian curvature).

Remark 2. In case of strictly negative Gaussian curvature, our argument in Appendix A shows that the graph contains a continuous family of line segments (and even an analytic family, if $f$ is analytic, cf. [38, Proof of Corollary 3]).

\section{Surfaces enveloped by a family of rotational cones, using a point model of the space of planes}

Now we come to the main topic of the paper: how to characterize surfaces enveloped by a one-parametric family of congruent cones? To minimize technicalities, we consider surfaces tangent to cones along curves rather than arbitrary envelopes of cones, 
and exclude certain positions of these curves. In this section we reduce the problem to the characterization of surfaces containing a special conic through each point, which is tractable by the methods already discussed.

\section{Motivation}

The motivation for using a plane-based approach is the following. A cone has just a one-parameter family of tangent planes $T(u)$. Moving the cone, seen as set of its tangent planes, under a generic smooth one-parameter motion, we obtain a two-parameter family of planes $T(u, v)$. These are precisely the tangent planes of the envelope!

One can convert the resulting (plane) representation of the envelope into its dual (point) variant by computing the intersection points

$$
\mathbf{r}(u, v)=T(u, v) \cap T_{u}(u, v) \cap T_{v}(u, v),
$$

where $T_{u}(u, v)$ and $T_{v}(u, v)$ are the planes with the equations obtained from the equation of $T(u, v)$ by taking partial derivatives. That is, if $T(u, v)$ has the equation

$$
n_{1}(u, v) x+n_{2}(u, v) y+n_{3}(u, v) z+h(u, v)=0,
$$

then $T_{u}(u, v)$ is given by

$$
\frac{\partial n_{1}(u, v)}{\partial u} x+\frac{\partial n_{2}(u, v)}{\partial u} y+\frac{\partial n_{3}(u, v)}{\partial u} z+\frac{\partial h(u, v)}{\partial u}=0 .
$$

This equation will not degenerate, as the intersections $T(u, v) \cap T_{u}(u, v)$ are the rulings of the cone. However, $T_{v}(u, v)$ may degenerate. Even all four partial derivatives with respect to $v$ may vanish simultaneously at particular points. Also, even if $T_{v}(u, v)$ is a well defined plane, the intersection (4) may be at infinity or be an entire straight line. For our purposes, it is not important to discuss all these cases and the corresponding properties of the generating motion. This is why we talked about a generic motion, which we want to be a one where (4) is always a well-defined point in $\mathbb{R}^{3}$ smoothly depending on $u, v$.

A few more informal remarks are in place: Note that we consider the whole unbounded moving cone and the possibly unbounded envelope. Also note that the envelope may consist of several parts and may have self-intersections. For example, when a rotational cylinder of radius $r$ moves so that its axis remains tangent to a generic space curve $c$, the envelope consists of two offset surfaces of the tangent developable of the curve $c$ and a pipe surface (the envelope of spheres of radius $r$, centered at c). By the way, the latter part of the envelope is useless for the CNC machining application we have in mind. We prefer to avoid envelopes in the precise statements of our results because this notion has slightly different definitions in the literature. (Sometimes this even leads to confusion: e.g., osculating circles of a generic curve are nested but all tangent to the curve; their envelope is the curve itself or empty depending on the choice of definition. In view of that notice that [38, Lemma 7] remains true for nested circles and should be applied in case (3) of the proof of Theorem 4 there.)

Anyway, converting the plane representation of the envelope into the point one is a postprocessing step and is not necessary for a characterization of these envelopes when we work in the space of planes.

\section{Definition of the point model}

Since geometric processing is easier in terms of points rather than planes, we apply a map that transforms planes to points and use a certain duality between plane and point coordinates. As we work with rotational cones, we use a transformation which allows us easily to recognize these cones in the point model. The right setting is that of Laguerre geometry ${ }^{1}$, the geometry of oriented planes [3, 9]. Laguerre geometry has already been useful in various applications in CAGD, see [13, 21, 22, 30, 31]. We try to give a concise, precise, and self-contained introduction to the subject; this is an update of [38, §2.3].

We introduce the following coordinates for planes in space. Let an oriented plane $P$ be given by the equation $n_{1} x+n_{2} y+$ $n_{3} z+h=0$, where $\left(n_{1}, n_{2}, n_{3}\right) \neq(0,0,-1)$ is the oriented unit normal to the plane and $|h|$ is the distance from the origin. The desired coordinates of the plane $P$ is the triple

$$
\left(\frac{n_{1}}{n_{3}+1}, \frac{n_{2}}{n_{3}+1}, \frac{h}{n_{3}+1}\right) .
$$

For the geometric considerations which lead to such coordinates, we refer to [31,32, 33]. To think geometrically, denote by $P^{i}$ the point with these coordinates, see Fig. 3. This correspondence between planes and points is called the isotropic model of Laguerre geometry; see [31, 32,33]. The simple non-Euclidean geometry in the point model, known as isotropic geometry, is treated in detail in [37].

To map an oriented surface $\Phi$ to the isotropic model, we consider the set $\Phi^{i}$ of points $P^{i}$, where $P$ runs through all oriented tangent planes to $\Phi$ with the oriented normals distinct from $(0,0,-1)$. Hereafter by an oriented surface we mean the image of a proper injective $C^{2}$ map of an open disk - or more generally of a smooth 2-manifold — into $\mathbb{R}^{3}$ with nondegenerate differential

\footnotetext{
${ }^{1}$ Another well-known assignment of points to planes is polarity with respect to the unit sphere. But it does not work that well because leads to "linear" functions on the sphere rather than on the plane, which are hard to deal with.
} 

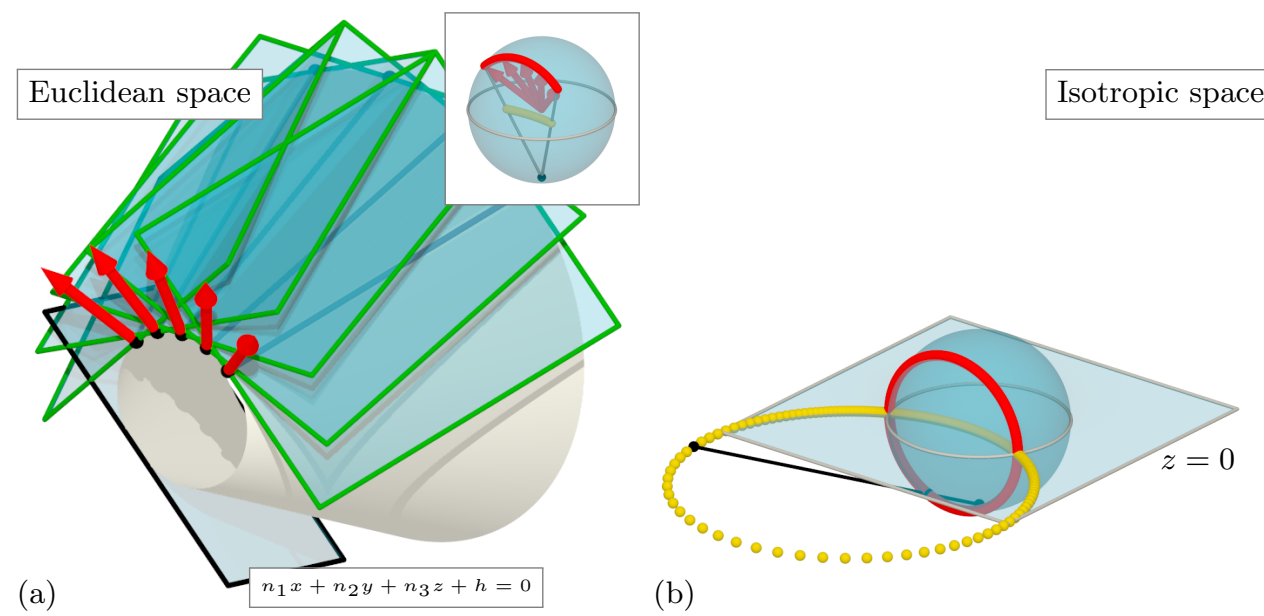

(b)

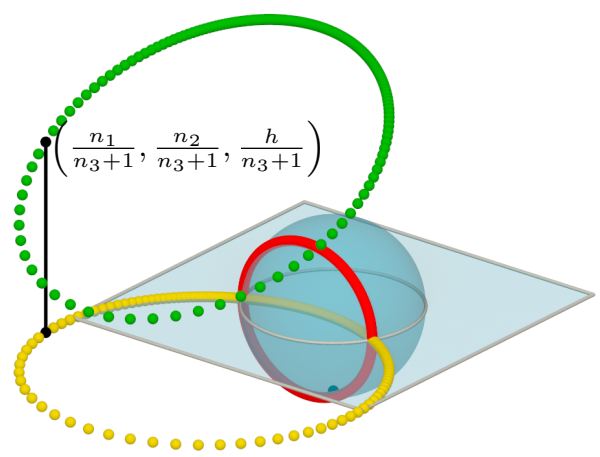

(c)

Figure 3: In the isotropic model of Laguerre geometry, planes appear as points and the tangent planes of a rotational cone are seen as special conics (isotropic circles): (a) A cone is considered as a one-parameter family of oriented planes and its normals (red) define a circle (only an arc is shown) on the Gaussian sphere (top-framed). (b) The Gaussian image (red circle) is projected from the south pole $(0,0,-1)$ to the $z=0$ plane to define the "top view" of the isotropic image (yellow). (d) The isotropic image of the cone (green conic), see Eq. (5).

at each point, equipped with oriented unit normals continuously depending on the point. For example, a sphere with center $\left(m_{1}, m_{2}, m_{3}\right)$, radius $R$, and inwards oriented normals is mapped to the rotational paraboloid (possibly degenerating to a plane)

$$
z=\frac{R+m_{3}}{2}\left(x^{2}+y^{2}\right)-m_{1} x-m_{2} y+\frac{R-m_{3}}{2} .
$$

The projection $(x, y, z) \mapsto(x, y, 0)$ of space onto the $x y$-plane is called top view. The top view of $\Phi^{i}$ is actually the stereographic projection of the Gaussian spherical image of $\Phi$ from the point $(0,0,-1)$ to the $x y$-plane, see Fig. 3(b). In particular, if the Gaussian curvature of $\Phi$ does not vanish, then $\Phi^{i}$ is locally a graph of a function.

By a cone we mean an oriented cone of revolution ${ }^{2}$. The opening angle $\theta$ of a cone is the angle between the axis and a ruling. A cone, viewed as the common tangent planes of two oriented spheres, is mapped to the common points of two paraboloids of form (6), i.e. a conic with the top view being a circle (or a parabola with the top view being a line). Such a conic is called a circle in isotropic geometry, or isotropic circle.

The shape of the top view can be obtained algebraically by eliminating $z$ from the system of two equations of form (6). But geometry gives more insight: the top view is the stereographic projection of the Gaussian spherical image of the cone, i.e., the projection of a circle of intrinsic radius $\pi / 2-\theta$ on the unit sphere; see Fig. 3. This leads to the following key observations.

Proposition 3. For a cone $C$ with the opening angle $\theta$ such that all the oriented unit normals are distinct from $(0,0,-1)$ the set $C^{i}$ is a conic satisfying the following condition:

$(\Theta)$ the top view of the conic is the stereographic projection of a circle of intrinsic radius $\pi / 2-\theta$ in the unit sphere (not passing through the projection center $(0,0,-1))$.

Proposition 4. Let $\Phi$ be an oriented surface in $\mathbb{R}^{3}$ with nowhere vanishing Gaussian curvature and the oriented unit normals distinct from $(0,0,-1)$. Then the following two conditions are equivalent:

- through each point of $\Phi$ there passes an oriented cone which is tangent to $\Phi$ along a continuous curve containing the point (not a ruling because the Gaussian curvature of $\Phi$ does not vanish), has the opening angle $\theta$, and has no oriented unit normals of the form $(0,0,-1)$;

- through each point of $\Phi^{i}$ there passes an arc of a conic contained in $\Phi^{i}$ and satisfying condition $(\Theta)$.

Practically the pieces of $\Phi$ where the Gaussian curvature vanishes are developable, hence trivially millable by a conical tool (possibly except the boundary of the set where the mean curvature vanishes as well). Thus in what follows we assume that the design surface $\Phi$ satisfies the following condition (likewise, this should be assumed throughout [38, §2.3]).

Condition $(*) \Phi$ is an oriented surface in $\mathbb{R}^{3}$ with nowhere vanishing Gaussian curvature such that all the oriented unit normals are distinct from $(0,0,-1)$, and $\Phi^{i}$ is the graph of a $C^{4}$ function $f: D \rightarrow \mathbb{R}$ in a disk $D \subset \mathbb{R}^{2}$.

This reduces the characterization of surfaces enveloped by a family of cones to the characterization of surfaces (actually graphs of functions) containing a special conic through each point. The latter is achieved in Section 5.

\footnotetext{
${ }^{2}$ To be precise, we exclude the vertex to get a smooth surface.
} 
Contact order in the space of planes

Recall that the derivation of the PDE for ruled surfaces in Section 3 has been based on expressing 3rd order contact between a straight line and a surface. We take a similar approach in the isotropic model of Laguerre geometry by looking at higher order contact between an isotropic circle and a surface. We now informally discuss the geometric meaning in the original design space. In the rest of Section 4 we omit the very technical formulations of the statements and proofs, because these subsections are not used in the proofs of main results, however, they help in understanding of the whole concept.

Definition 5. Let $f$ be a $C^{n}$ function in a disk $D \subset \mathbb{R}^{2}$. Let $(x(t), y(t), z(t))$, where $t$ runs through an interval $I$, be a $C^{n}$ curve such that $(\dot{x}(t), \dot{y}(t)) \neq 0$ for each $t \in I$. We say that the curve has contact of order $n$ with the graph of $f$ at $t=0$, if

$$
\frac{z(t)-f(x(t), y(t))}{t^{n}} \rightarrow 0 \text { as } t \rightarrow 0 .
$$

In particular, a curve intersecting the graph for $t=0$ has contact of order 0 ; a curve tangent to the graph for $t=0$ has contact of order 1, etc.; a curve fully contained in the graph has contact of infinite order.

Likewise, two curves $c_{1}, c_{2}$ have contact of order $n$ at a common point if there are regular parameterizations $c_{1}(t), c_{2}(t)$ of these curves that agree for some $t=t_{0}$ in function value and derivatives up to the $n$-th order. A totally analogous definition holds for two surfaces. Contact order $n$ between a curve $c$ and surface $\Phi$, as given in the above definition, can also be defined as follows: the surface $\Phi$ contains a regular smooth curve $c_{1}$ which has $n$-th order contact with the curve $c$. This curve $c_{1} \subset \Phi$ is not uniquely determined. If there is one such curve $c_{1}$, there are infinitely many other curves in $\Phi$ which verify $n$-th order contact with $c$. For example, consider a tangent line $c$ at an elliptic point of $\Phi$ and a pencil of planes that contain $c$. Then each plane of the pencil intersects $\Phi$ in a curve $c_{1}$ that each has the first order contact with $c$.

Consider two regularly parametrized curves $C_{1}^{i}(t), C_{2}^{i}(t)$ in the isotropic model which have contact of order $n \geq 1$ at some common point $C_{1}^{i}\left(t_{0}\right)=C_{2}^{i}\left(t_{0}\right)$. The curves as point sets correspond to plane families in design space. Their envelopes are two developable surfaces $C_{1}, C_{2}$. It is not hard to show that these developable surfaces have a common ruling and contact of order $n$ at each point of the ruling (see [34]). Let us now assume that we have contact of order $n$ between a curve $C^{i}$ and a surface $\Phi^{i}$ in the isotropic model. In design space, this corresponds to a developable surface $C$ and a surface $\Phi$. However, $C$ and $\Phi$ do not have contact of order $n$ if we view these surfaces as point sets: For instance, if a cone $C$ is tangent to a sphere $\Phi$ along a circle, then $C^{i}$ is contained in $\Phi^{i}$, hence has contact of arbitrarily high order. But the rulings of $C$ have contact order just 1 with the sphere $\Phi$. We have to view $C$ and $\Phi$ as plane sets. This means that there exist (in fact, infinitely many) tangent developable surfaces of $\Phi$ which have $n$-th order contact with $C$. We illustrate this in the following at hand of examples that are very relevant for our setting.

\section{Second order contact}

Let $C^{i}$ be a curve in the isotropic model. At each point $C^{i}\left(t_{0}\right)$, the curve has an osculating isotropic circle $C_{o}^{i}$. It has 2 nd order contact with $C^{i}$ at $C^{i}\left(t_{0}\right)$, lies in the osculating plane and its top view is the Euclidean osculating circle of the top view of $C^{i}$, see Fig. 4. In the original space, $C^{i}$ corresponds to a set of planes which envelope a certain developable surface $C$. The osculating isotropic circle corresponds to a cone of revolution $C_{o}$. It has 2nd order contact with the developable surface $C$ along an entire common ruling and is called its osculating cone along that ruling. The vertex of the cone lies on the (singular) regression curve of $C$; see e.g. [34, Theorem 6.1.4].

Assume now that the curve $C^{i}$ lies on some surface $\Phi^{i}$. An isotropic osculating circle $C_{o}^{i}$ of $C^{i}$ has 2 nd order contact with $\Phi^{i}$. Mapping back to design space, we obtain a developable surface $C$ which is tangent to a surface $\Phi$ along some curve. The isotropic circle $C_{o}^{i}$ corresponds to an osculating cone $C_{o}$ of $C$. That cone does not have 2nd order contact with the surface, if one views the cone as a point set. However, the cone has 2 nd order contact as a set of planes. This means that there exist tangent developable surfaces of $\Phi$ which have 2 nd order contact with $C_{o}$. Among those tangent developables we can take a special one, namely the cone $C_{1}$ (not necessarily of revolution) which shares the vertex $v$ with $C_{o}$. It is enveloped by all tangent planes of $\Phi$ that pass through the point $v$ (or, equivalently, are tangent to the sphere with center $v$ and radius zero). (In general position, $v$ does not belong to the surface $\Phi$, and in particular $v^{i}$ (isotropic sphere) is transversal to $\Phi^{i}$ at the contact point of $C^{i}$ and $C_{o}^{i}$.) The intersection of $C_{1}$ with a plane $P$ is the contour of $\Phi$ under a central projection from the point $v$ onto the plane $P$. For example, if we take an image plane orthogonal to the axis of $C_{o}$, its intersection with $C_{o}$ is a circle. This circle is the osculating circle of the contour of $\Phi$ for projection from $v$ onto $P$.

Mannheim sphere. Like in Euclidean geometry, there is a Meusnier's theorem in isotropic geometry: Given a surface $\Phi^{i}$, and a point $P^{i} \in \Phi^{i}$ with a surface tangent $T^{i}$. Then, the osculating isotropic circles of all curves $C^{i} \subset \Phi^{i}$ which pass through $P^{i}$ with tangent $T^{i}$, lie in an isotropic sphere. Mapping back to design space, we obtain Mannheim's theorem [23]: The osculating cones of all developable surfaces $C$ which are tangent to a given surface $\Phi$ and have a common ruling $R$ (tangent to $\Phi$ ), are tangentially circumscribed to a sphere (Mannheim sphere). This gives an overview of all cones which have 2 nd order contact (as plane sets) with a given surface at a fixed point and allows one to apply additional constraints, for example, on the opening angle of the cone. 


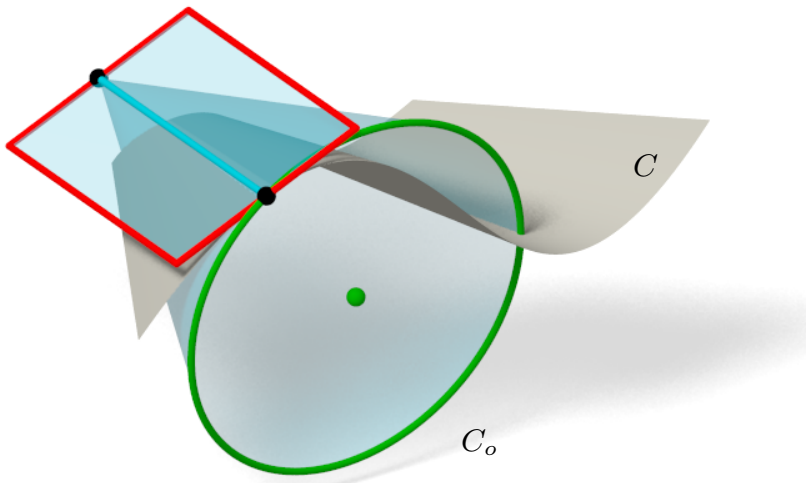

(a)

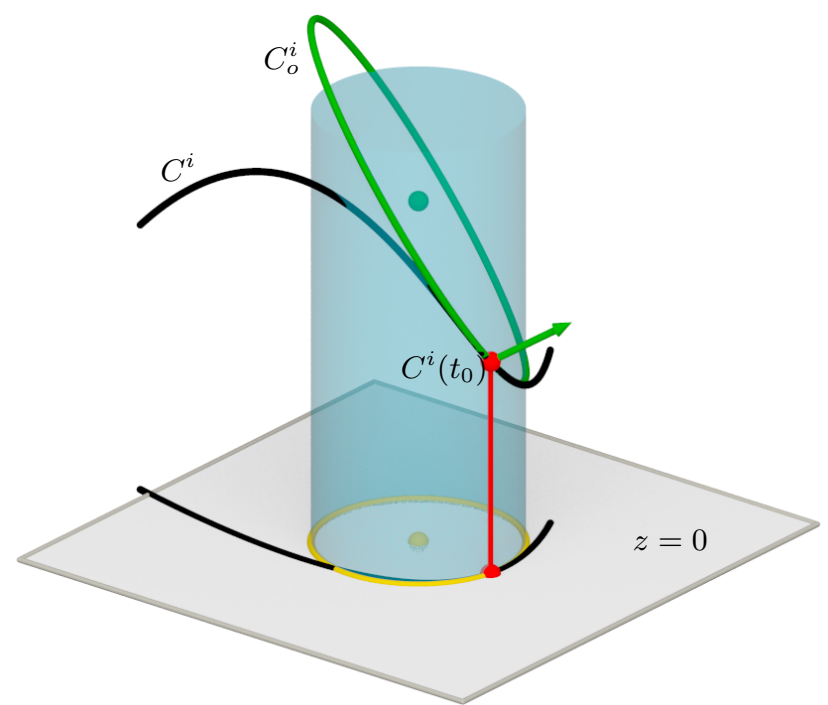

(b)

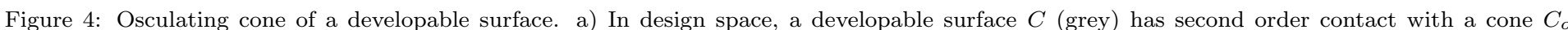

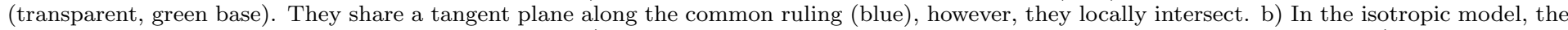

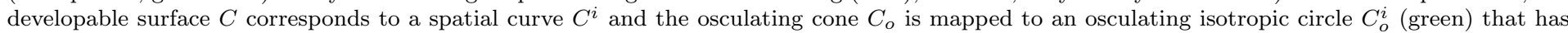
second order contact with $C^{i}$. The top view is a circle (yellow) in the plane $z=0$ that osculates the projection of $C^{i}$.

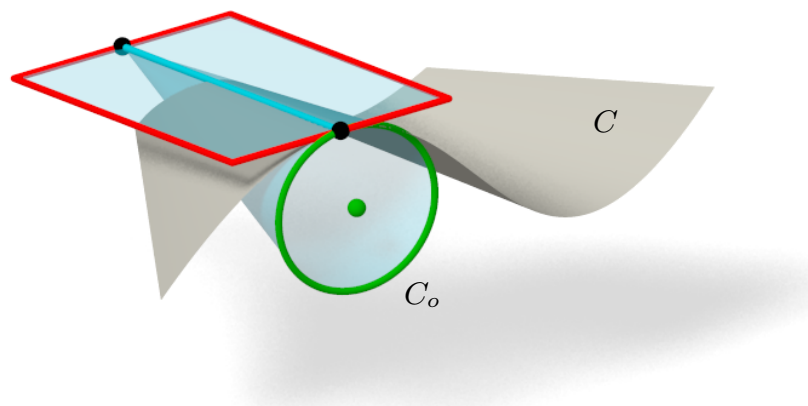

(a)

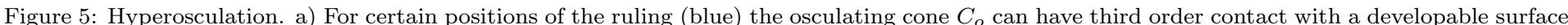

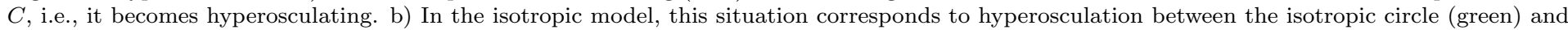
the spatial curve $C^{i}$ that represents the developable surface $C$. In the top view, the isotropic circle (yellow) hyperosculates the projection of $C^{i}$.

\section{Third order contact}

In Euclidean geometry, there are results on circles which have 3rd order contact with a given surface at a given point. These have even been proposed for CNC machining with a cylindrical cutter since the bottom circle of the cutter will actually generate the shape and thus 3rd order contact leads to a good surface finish, at least in theory $[46,47,50]$. In fact, 2nd order contact is in general not enough because an osculating circle of a surface will locally change the side of the surface and thus cause gauging. That is, if the osculating circle is parametrized by an arc-length parameter $h$ so that $h=0$ corresponds to the contact point, then the 2nd order contact means that the signed distance to the surface is $\sim h^{3}$ and therefore changes its sign in a neighborhood of the contact point. Consequently, there is local penetration (called gauging). This local interference is not present for 3rd order contact (aka hyperosculation) because the error is $\sim h^{4}$ and therefore, locally, the hyperosculating circle lies on one side of the reference surface. In practice, it is hard to find a path which leads the cutter in such a way that the bottom circle stays in 3rd order contact with the surface. However, knowing that 3rd order contact is a limit of a double contact, Kim et al. [50] used hyperosculating circles for initializing an optimization algorithm which leads the cutter such that it has a double contact with the target surface.

We derive an analogous characterization of hyperosculation in isotropic geometry. We look for hyperosculating isotropic circles; in the original design space, they correspond to rotational cones that have 3rd order contact with the surface. Again, this 3rd order contact is not in the sense of point sets, but in the (dual) sense of plane sets, see Fig. 5. We expect that 

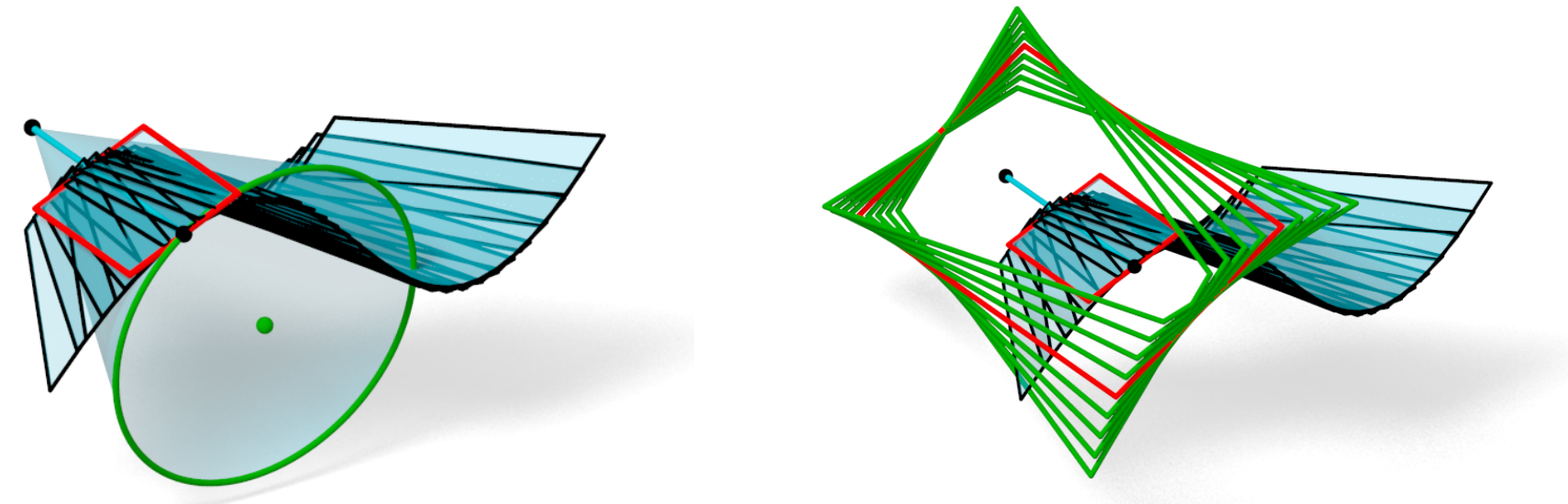

(a)

(b)

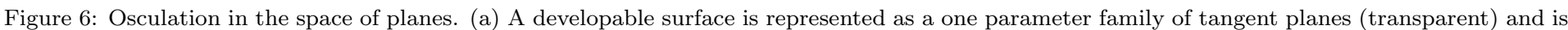

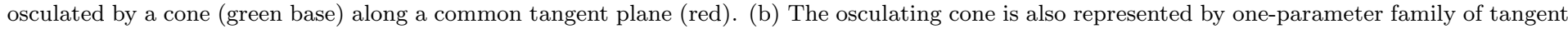
planes (green) and the two families osculate at the red plane. Observe that the two red planes are identical.

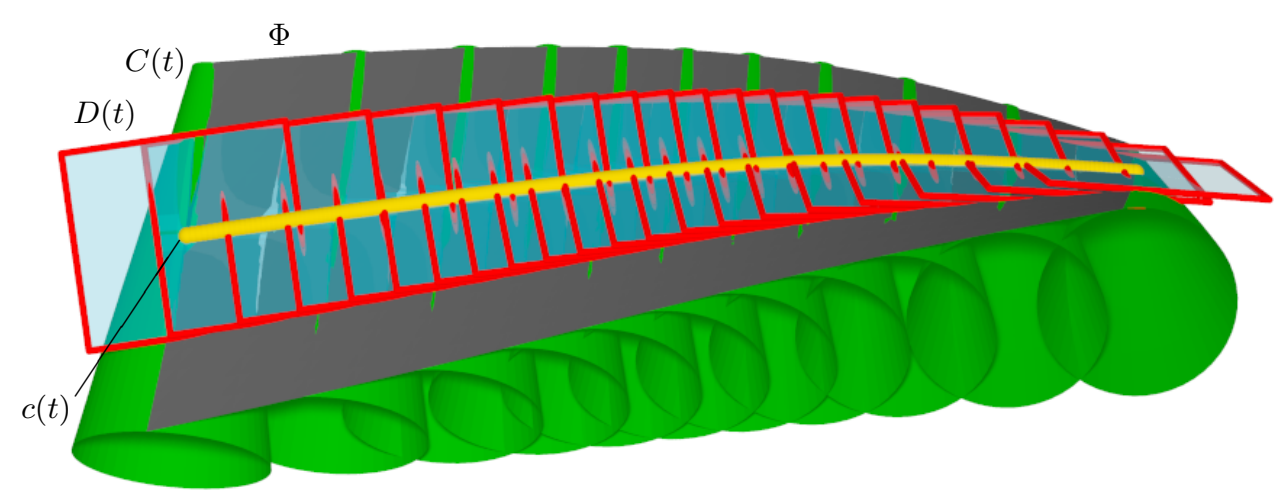

(a)

(b)

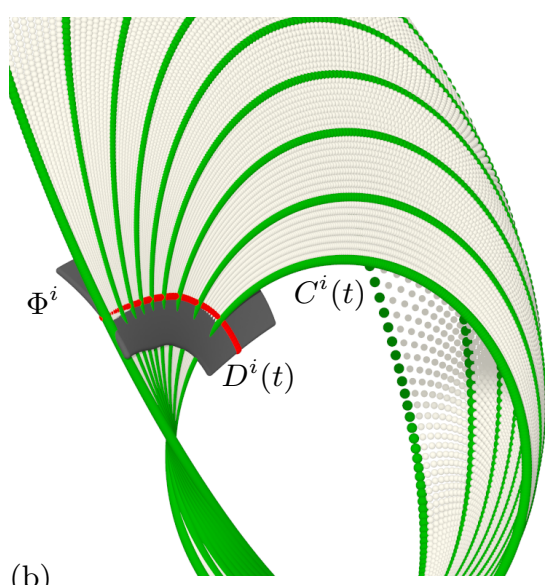

Figure 7: Approximation of a general surface. (a) A reference surface $\Phi$ (grey) is approximated by an envelope of a moving cone (green) in the neighborhood of the contact curve $c(t)$ (yellow). Along this curve, they share a one-parameter family of tangent planes $D(t)$ (red). (b) In the isotropic space, the tangent planes are mapped to a curve $D^{i}(t)$ (red) that lies on the isotropic image $\Phi^{i}$ of the surface. The tangent planes of each cone are mapped to an isotropic circle (green) intersecting the curve $D^{i}$ transversely.

hyperosculating cones will have CNC applications in the original design space as they fit better (up to higher order) the input surface. Moreover, near such positions one can find doubly tangent cones. This is not exploited for CNC machining in the present paper, but could be the topic of future research.

\section{Higher order contact between a surface and an envelope of cones}

Let us now return to the application in CNC machining with a conical tool. We want to approximate a given surface $\Phi$ with nowhere vanishing Gaussian curvature (design surface) by an envelope of a moving rotational cone (tool). We claim that having contact of order $n$ between tool positions and $\Phi$ in the space of planes guarantees contact of order $n$ between $\Phi$ and the envelope surface $\Psi$ generated by the moving tool, and vice versa. We only have to make sure that the tool is always moved into an appropriate direction. We will now show that almost all directions are appropriate, except for the one which is conjugate to the ruling of the tool at the contact point.

To explain that, let us first have a look into the isotropic model. There, the tool positions $C(t)$ appear as isotropic circles $C^{i}(t)$ which have contact of order $n$ with $\Phi^{i}$. As long as the curve of contact points $D^{i}(t)$ of these circles is transversal to the circles, we have contact of order $n$ between the surface $\Psi^{i}$ generated by the isotropic circles (envelope in the isotropic model) and $\Phi^{i}$, see Fig. 7. Mapping back to $\mathbb{R}^{3}$, we obtain contact of order $n$ between the surface $\Phi$ and the envelope $\Psi$. We just have to clarify how to recognize the mentioned transversality in the isotropic model directly in design space $\mathbb{R}^{3}$. The contact curve $D^{i}$ corresponds to a developable surface $D \subset \mathbb{R}^{3}$ which is the envelope of the common tangent planes $D(t)$ of the moving tool and $\Phi$ at the cutter contact points. In other words, this developable surface $D$ is tangent to $\Phi$ along the set of cutter contact points $c(t)$, which form a curve $c \subset \Phi$. The mentioned transversality in the isotropic model means that none of the isotropic circles $C^{i}(t)$ is tangent to the contact curve $D^{i}$. As we have already discussed, two curves in the isotropic model share 
Proposition 6. Each conic satisfying condition $(\Theta)$ can be parametrized as

$$
\left\{\begin{array}{l}
x(t)=x+v \sin t+u(1-\cos t) \\
y(t)=y-u \sin t+v(1-\cos t) \\
z(t)=z+a \sin t+b(1-\cos t)
\end{array}\right.
$$

where $a, b, u, v, x, y, z \in \mathbb{R}$ satisfy

$$
\left(x^{2}+y^{2}+1+2 x u+2 y v\right)^{2}-4 \tan ^{2} \theta\left(u^{2}+v^{2}\right)=0 .
$$

a common point and tangent, if the corresponding developable surfaces in Euclidean space share a common tangent plane and

ling. Hence, the cone rulings $r_{C}(t)$ through the contact points $c(t)$ have to be different from the rulings $r_{D}(t)$ of $D$.

Essentially, we are still in the space of planes. To get information about an appropriate contact curve $c(t)$, we recall a lassical result: Given a curve $c$ on a surface $\Phi$, the developable surface $D$ which is tangent to $\Phi$ along $c$ has rulings $r_{D}(t)$ are conjugate to the tangents of $c$. This conjugacy is with respect to the osculating quadric of $\Phi$ at the contact point 作 in the directions of $\mathbf{v}$ and $\mathbf{w}$. Then the tangent lines of the Dupin indicatrix at the endpoints of the first diameter are parallel o the second one (and vice versa); see [34, page 262 and 334] for more details. So, the rulings $r_{C}(t)$ and $r_{D}(t)$ are different, if cirections with respect to $\Phi$ are different. Hence, the tangent of the cutter contact curve $c$ has to be different njugate direction to obtain the widest machined strips.

Note that we are interested here in contact of order $n \geq 2$, which essentially means $n=2$ or $n=3$. There, the envelope of the moving tool and the target surface $\Phi$ share the conjugacy relation at the contact points. The conjugate direction of the cone ruling $r_{C}(t)$ at the contact point with respect to the envelope of the cones is the tangent to the characteristic (since the cone is the tangent developable of the envelope along the characteristic). Hence, an appropriate direction of the tool movement which is transversal to the characteristic. This is exactly what one would expect. But note that when we want to plan

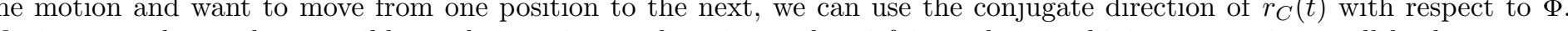
appropriate cone position. For actual machining, osculation $(n=2)$ will lead to overcutting. However, one can use a motion with an osculating envelope as a guide and work with a slightly smaller cutting tool to avoid overcutting. Recall once again that the discussion throughout this section is valid under certain technical assumptions like general position and non-vanishing

\section{Surfaces containing a special conic through each point}

In this section we characterize the surfaces containing a conic satisfying condition $(\Theta)$ through each point (Theorem 10 below). This is required for the main result in Section 6. The characterization is similar to that of ruled surfaces in Section 3 . We the graph of a smooth function $f$. The conics on the graph are parametrized by trigonometric functions. Differentiation with respect to the parameter gives a system of algebraic equations on the tangential direction to the top view of the conic at a given point. Solvability of the system is the required condition on $f$.

\section{Conics parametrization}

where a, b, $, u, x, y, z \in \mathbb{R}$ satisfy

Proof of Proposition 6. Let $(x, y, z)$ be a point on the conic and $(x+u, y+v)$ be the center of the top-view circle. Clearly, then the circle is parametrized by $x(t)$ and $y(t)$ from (7). Since a conic is a planar curve, $z(t)$ must be a linear function in $x(t)$ and $y(t)$, and we arrive at $(7)$ for some $a, b \in \mathbb{R}$.

Now turn to condition $(\Theta)$. Let $A$ and $B$ be the points of the top-view circle which are the closest and the furthest from the origin $O$ (or just opposite points, if $O$ is the center). The inverse stereographic projection of the circle from the point $S=(0,0,-1)$ is a circle of intrinsic radius $\angle A S B$ in the unit sphere. Thus

$$
\pm \cot \theta=\tan A S B=\frac{\tan B S O \mp \tan A S O}{1 \pm \tan A S O \tan B S O}=\frac{O B \mp O A}{1 \pm O A \cdot O B}=\frac{2 \sqrt{u^{2}+v^{2}}}{1+(x+u)^{2}+(y+v)^{2}-u^{2}-v^{2}}
$$

where the choice of sign in the left-hand side depends on if $\angle A S B$ is acute or obtuse, and the other signs depend on if $O$ is outside or inside the top-view circle. We arrive at (8).

\section{Derivation of the system}

Let us derive PDEs for functions whose graphs contain a conic satisfying condition $(\Theta)$ through each point. We are not actually using that the entire conic is contained in the graph; a sufficiently high contact suffices. 


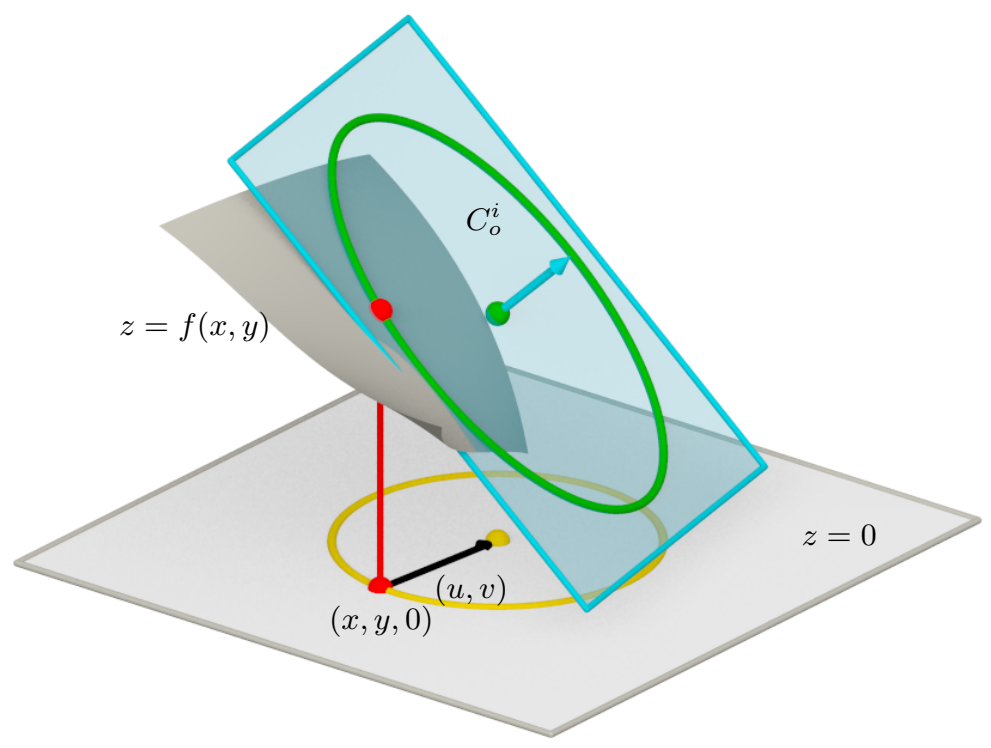

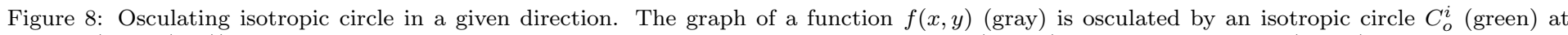

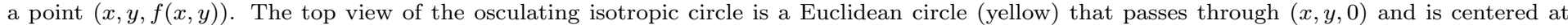
$(x+u, y+v, 0)$. The constraints for osculation between $f$ and $C_{o}^{i}$ are given in Eq. (9).

322 Example 7. The graph of the function $f(x, y)=\frac{y^{2}}{x^{2}+y^{2}}$, where $(x, y) \neq(0,0)$, is covered by a 2 -dimensional family of conics $(7)$ with $u=-\frac{x}{2}, v=-\frac{y}{2}, z=\frac{y^{2}}{x^{2}+y^{2}}, a=\frac{x y}{x^{2}+y^{2}}, b=\frac{x^{2}-y^{2}}{2\left(x^{2}+y^{2}\right)}$. The ones with $x^{2}+y^{2}=\cot ^{2} \theta$ satisfy condition $(\Theta)$. We return to this example in Section 8.

Proposition 8. Conic (7) has contact of order 2 with the graph of $f$ ("osculation"), if and only if

$$
\left\{\begin{array}{l}
z=f(x, y) \\
a=f_{x} v-f_{y} u \\
b=f_{x} u+f_{y} v+f_{x x} v^{2}-2 f_{x y} u v+f_{y y} u^{2} .
\end{array}\right.
$$

The contact order is 3 ("hyperosculation"), if and only if in addition

$$
f_{x x x} v^{3}-3 f_{x x y} v^{2} u+3 f_{x y y} v u^{2}-f_{y y y} u^{3}+3\left(f_{x x}-f_{y y}\right) u v+3 f_{x y}\left(v^{2}-u^{2}\right)=0 .
$$

The contact order is 4 , if and only if in addition

$$
\begin{array}{r}
f_{x x x x} v^{4}-4 f_{x x x y} v^{3} u+6 f_{x x y y} v^{2} u^{2}-4 f_{x y y y} v u^{3}+f_{y y y y} u^{4} \\
+6 u v^{2} f_{x x x}+6 v\left(v^{2}-2 u^{2}\right) f_{x x y}+6 u\left(u^{2}-2 v^{2}\right) f_{x y y} \\
+6 u^{2} v f_{y y y} \\
+3\left(u^{2}-v^{2}\right)\left(f_{x x}-f_{y y}\right)+12 u v f_{x y}=0 .
\end{array}
$$

Proof of Proposition 8. The proof is by consecutive differentiation of $z(t)-f(x(t), y(t))$ with respect to $t$ and evaluating at $t=0$. For instance, the second derivative is

$$
\begin{aligned}
& -a \sin t+b \cos t-f_{x}(u \cos t-v \sin t)-f_{y}(v \cos t+u \sin t) \\
& \quad-f_{x x}(v \cos t+u \sin t)^{2}-2 f_{x y}(v \cos t+u \sin t)(v \sin t-u \cos t)-f_{y y}(v \sin t-u \cos t)^{2}
\end{aligned}
$$

For contact of order 2 , this must vanish at $t=0$, which gives the third equation of (9).

Equation (9) is an expression that links together the point $(x, y, z)$ in the isotropic space, the center $(x+u, y+v, 0)$ of the top-view circle in the plane $z=0$, two parameters $a$ and $b$ that control the inclination of the plane that contains the isotropic circle, and the derivatives of the function $f$; see Fig. 8 . The remaining equations (10)-(11) together with (8) give a nontrivial restriction on the function $f$ itself.

Corollary 9. Let $f$ be a $C^{4}$ function in a disk $D \subset \mathbb{R}^{2}$. If through each point of the surface $z=f(x, y)$ there passes an arc of a conic satisfying condition $(\Theta)$ and completely contained in the surface, then for each $(x, y) \in D$ three equations (8),(10),(11) have a common real solution $(u, v)$. 

solution $(u, v)$ nowhere satisfying the equation (where the left-hand side is the Jacobian of (8) and (10))

$$
f_{x x x} v^{2} \tilde{u}+f_{x x y} v(v \tilde{v}-2 u \tilde{u})+f_{x y y} u(u \tilde{u}-2 v \tilde{v})+f_{y y y} u^{2} \tilde{v}+\left(f_{x x}-f_{y y}\right)(u \tilde{u}-v \tilde{v})+2 f_{x y}(u \tilde{v}+v \tilde{u})=0
$$

where

$$
\tilde{u}=x\left(x^{2}+y^{2}+1+2 x u+2 y v\right)-4 u \tan ^{2} \theta, \quad \tilde{v}=y\left(x^{2}+y^{2}+1+2 x u+2 y v\right)-4 v \tan ^{2} \theta .
$$

The restriction that the conic continuously depends on the point seems inessential; it is imposed to bypass technical issues discussed in Appendix A. But dropping the restriction that the conic is nonmultiple would require new ideas, just like developable surfaces require special treatment in characterization of ruled surfaces in Appendix A.

Problem 12. Prove the reciprocal assertion in Corollary 9 in the case when (14) holds identically, i.e. the conic is multiple. Is it true that in this case the surface $z=f(x, y)$ is the envelope of a one-parametric family of rotational paraboloids (6) such that each characteristic is a conic satisfying condition $(\Theta)$ ?

\section{Surfaces enveloped by a family of rotational cones: conclusion}

Now we use the results of the previous two sections to complete the characterization of surfaces enveloped by a one-parametric family of congruent cones (Theorem 13 below). Then we show how to construct $\Phi^{i}$ from $\Phi$ and vice versa. Finally we show how to reconstruct the positions of cones in the enveloping family.

\section{Characterization of surfaces enveloped by a family of cones}

We summarize now the previous results. From Proposition 4, Corollary 9, and Theorem 10 together we get the following characterization.

Theorem 13 (characterization of surfaces enveloped by a family of cones). Assume (*).

If through each point of $\Phi$ there passes a cone which is tangent to $\Phi$ along a curve (containing the point), has the opening angle $\theta$, and has no oriented unit normals equal to $(0,0,-1)$, then for each $(x, y) \in D$ three equations (8), (10), (11) have a common nonzero real solution $(u, v)$.

Conversely, if for each $(x, y) \in D$ three equations (8), (10), (11) have a common real solution (u,v) continuously depending on $(x, y)$ and nowhere satisfying (14), then through each point of $\Phi$ there passes a cone which is tangent to $\Phi$ along a continuous curve (containing the point) and has the opening angle $\theta$. 
Proposition 15. (Cf. [32, Corollary 2]) Assume $\left({ }^{*}\right)$. Let $\left(n_{1}, n_{2}, n_{3}\right)$ be the oriented unit normal at a point $\left(r_{1}, r_{2}, r_{3}\right)$ of $\Phi$. Then the function $f$ and its derivatives are given by

$$
\begin{aligned}
f\left(\frac{n_{1}}{n_{3}+1}, \frac{n_{2}}{n_{3}+1}\right) & =-\frac{n_{1} r_{1}+n_{2} r_{2}+n_{3} r_{3}}{n_{3}+1}, \\
f_{x}\left(\frac{n_{1}}{n_{3}+1}, \frac{n_{2}}{n_{3}+1}\right) & =\frac{n_{1} r_{3}}{n_{3}+1}-r_{1}, \\
f_{y}\left(\frac{n_{1}}{n_{3}+1}, \frac{n_{2}}{n_{3}+1}\right) & =\frac{n_{2} r_{3}}{n_{3}+1}-r_{2} .
\end{aligned}
$$

Conversely, given the function $f$, the surface $\Phi$ can be parametrized as follows:

$$
\mathbf{r}(x, y)=\frac{1}{x^{2}+y^{2}+1}\left(\begin{array}{c}
\left(x^{2}-y^{2}-1\right) f_{x}+2 x y f_{y}-2 x f \\
\left(y^{2}-x^{2}-1\right) f_{y}+2 x y f_{x}-2 y f \\
2 x f_{x}+2 y f_{y}-2 f
\end{array}\right) .
$$

Here the point $\mathbf{r}(x, y)$ is the tangency point of $\Phi$ and the plane $P$ such that $P^{i}=(x, y, f(x, y))$.

In what follows we use the formula for the inverse stereographic projection from $(0,0,-1)$ :

$$
\mathbf{n}(x, y)=\frac{\left(2 x, 2 y, 1-x^{2}-y^{2}\right)}{x^{2}+y^{2}+1} .
$$

Proof. The oriented tangent plane $P$ to $\Phi$ at the point $\left(r_{1}, r_{2}, r_{3}\right)$ is given by $n_{1} x+n_{2} y+n_{3} z-n_{1} r_{1}-n_{2} r_{2}-n_{3} r_{3}=0$. By the definition of $P^{i}$ we get $(16)$. Now let $(x, y)=\left(\frac{n_{1}}{n_{3}+1}, \frac{n_{2}}{n_{3}+1}\right)$ be the stereographic projection of $\left(n_{1}, n_{2}, n_{3}\right)$. Then $\frac{n_{3}}{n_{3}+1}=\frac{1}{2}\left(1-x^{2}-y^{2}\right)$ by (20). Substituting these expressions into (16), differentiating with respect to $x$, and using the condition $n_{1} \frac{\partial}{\partial x} r_{1}+n_{2} \frac{\partial}{\partial x} r_{2}+n_{3} \frac{\partial}{\partial x} r_{3}=0$ that $\left(n_{1}, n_{2}, n_{3}\right)$ is normal to $\Phi$, we get (17). Analogously we get (18). Solving (16)-(18) as a linear system in $r_{1}, r_{2}, r_{3}$ we get (19).

\section{Reconstruction of the cones}

To determine the position of a cone $C$ with a given opening angle and tangent to a given surface at a given point, it suffices to identify the position of the vertex and the side of the tangent plane which the cone borders upon at the tangency point (i.e. the halfspace containing a small neighborhood of the tangency point on the cone).

The vertex is reconstructed from the conic $C^{i}$ as follows.

Proposition 16. Let $C$ be the cone such that the conic $C^{i}$ is parametrized by $(7)$; then the vertex of $C$ is

$$
\begin{aligned}
& \mathbf{m}(x, y)=\frac{1}{\left(u^{2}+v^{2}\right)\left(x^{2}+y^{2}+1+2 u x+2 v y\right)} \\
& \times\left(\begin{array}{c}
\left(x^{2}-y^{2}-1\right)(a v+b u)+2 x y(b v-a u)-2\left(u^{2}+v^{2}\right)(u z+x z+a y) \\
\left(y^{2}-x^{2}-1\right)(b v-a u)+2 x y(a v+b u)-2\left(u^{2}+v^{2}\right)(v z+y z-a x) \\
2 x(a v+b u)+2 y(b v-a u)-2\left(u^{2}+v^{2}\right) z
\end{array}\right) .
\end{aligned}
$$

Proof. Let $\left(m_{1}, m_{2}, m_{3}\right)$ be the vertex of $C$. Then conic (7) must be contained in surface (6) with $R=0$. Substitute (7) into (6). Consecutively differentiating the left-hand side of (6) two times with respect to $t$, substituting $\dot{x}(0)=v, \dot{y}(0)=-u$, $\ddot{x}(0)=u, \ddot{y}(0)=v, R=0$, and solving the resulting system of 3 linear equations in $m_{1}, m_{2}, m_{3}$, we get $(21)$. 
The side which the cone $C$ borders the tangent plane $P$ upon at the point $\mathbf{r}$ can be identified as follows.

Proposition 17. Let a plane $P$ and a cone $C$ be such that $P^{i}=(x, y, z)$ and the conic $C^{i}$ is parametrized by (7). In particular, the vector $\mathbf{n}(x, y)$ given by (20) is normal to $P$. Let $\mathbf{m}$ be the vertex of $C$ and $\mathbf{r}$ be a tangency point of $C$ and $P$. Take any point $\left(x^{\prime}, y^{\prime}\right)$ in the top view of $C^{i}$ distinct from $(x, y) ;$ e.g., $\left(x^{\prime}, y^{\prime}\right)=(x+2 u, y+2 v)$. Then $C$ borders upon $P$ at $\mathbf{r}$ from the side of the halfspace containing

$$
\begin{cases}\mathbf{n}(x, y), & \text { if } \mathbf{n}\left(x^{\prime}, y^{\prime}\right) \cdot(\mathbf{r}-\mathbf{m})>0 \\ -\mathbf{n}(x, y), & \text { if } \mathbf{n}\left(x^{\prime}, y^{\prime}\right) \cdot(\mathbf{r}-\mathbf{m})<0\end{cases}
$$

Proof. For $\left(x^{\prime}, y^{\prime}\right)=(x+2 u, y+2 v)$ cut the cone $C$ by the plane passing through $\mathbf{m}$ and $\mathbf{r}$ and being parallel to $\mathbf{n}(x, y)$. Then the plane is parallel to the vector $\mathbf{n}\left(x^{\prime}, y^{\prime}\right)$ as well, and the proposition reduces to an obvious planar problem. For other $\left(x^{\prime}, y^{\prime}\right) \neq(x, y)$ the proposition follows by the continuity.

\section{Envelopes of congruent rotational cylinders}

Cylinders are a limit case of cones, but this limit is not straightforward. This is so, since the limit of cones with a constant opening angle are cones with vanishing opening angle, i.e., rotational cylinders. However, these cylinders need not be congruent. Hence, we now discuss envelopes of congruent rotational cylinders, i.e. offsets of ruled surfaces, which appear in flank CNC machining with a cylindrical tool.

The derivation of the PDE is analogous to Sections 4-5. Passing to the isotropic model, we reduce the characterization of surfaces in question to the characterization of surfaces containing a special conic through each point. We parametrize the conic by trigonometric functions and identify the particular conditions on the conic. Differentiation with respect to the parameter gives the required PDE.

Proposition 18. Assume $\left(^{*}\right)$. Through each point of $\Phi$ there passes an oriented cylinder of fixed radius $R$ which is tangent to $\Phi$ along a continuous curve (containing the point), has inwards oriented normals, and the axis nonparallel to the plane $z=0$, if and only if through each point of the surface $\Phi^{i}$ there passes an arc of a conic satisfying the following condition:

$(R)$ the top view of the conic is the stereographic projection of a great circle (not passing through the projection center $(0,0,-1))$, and the plane of the conic passes through the point $(0,0, R)$.

Remark 19. A similar propositions holds for a cylinder with outwards oriented normals, only $(0,0, R)$ is replaced by $(0,0,-R)$.

Proof. Let $C$ be an oriented cylinder of radius $R$ with inwards oriented normals and the axis nonparallel to the plane $z=0$. The oriented tangent planes to $C$ are the common oriented tangent planes of some two oriented spheres $S_{1}$ and $S_{2}$ of radius $R$ with inwards oriented normals. Then $C^{i}$ is the intersection of $S_{1}^{i}$ and $S_{2}^{i}$. Assume that $S_{1}$ is contained in the halfspace $z \leq 0$ and tangent to the plane $z=0$. Then $S_{2}^{i}$ is a paraboloid of form (6), and $S_{1}^{i}$ is a plane. Hence $C^{i}$ is a conic. Since the oriented sphere $S_{1}$ is tangent to the oriented plane $P$ given by $z=-2 R$ with the normal $(0,0,1)$, by (5) it follows that the plane $S_{1}^{i}$ of the conic passes through the point $P^{i}=(0,0, R)$. The top view of $C^{i}$ is the stereographic projection of the Gaussian spherical image of $C$, i.e., the projection of a great circle. Now if $C$ is tangent to $\Phi$ along a curve (which cannot be a ruling because by (*) $\Phi$ has nonvanishing Gaussian curvature), then $C^{i}$ is contained in $\Phi^{i}$. The proof of the reciprocal implication is analogous.

Proposition 20. Consider conic (7), where $a, b, z$ are given by (9) for some $C^{2}$ function $f: D \rightarrow \mathbb{R}$. Then the conic satisfies condition $(R)$, if and only if the following two equations hold:

$$
\begin{gathered}
x^{2}+y^{2}+1+2 x u+2 y v=0 \\
2\left(u^{2}+v^{2}\right)\left(f-x f_{x}-y f_{y}-R\right)+\left(x^{2}+y^{2}+1\right)\left(f_{x x} v^{2}-2 f_{x y} u v+f_{y y} u^{2}\right)=0 .
\end{gathered}
$$

Proof. Since a great circle in the unit sphere has intrinsic radius $\pi / 2$, substituting $\theta=0$ into (8), we get (22). If the plane of conic (7) passes through $(0,0, R)$, we get $z(t)=A x(t)+B y(t)+R$ for some constants $A, B \in \mathbb{R}$. Hence

$$
\left\{\begin{array}{l}
z=A x+B y+R, \\
a=A v-B u \\
b=A u+B v
\end{array}\right.
$$

The latter two equations in $A$ and $B$ are linearly independent because $(u, v) \neq(0,0)$ by $(22)$. Thus the system has a solution $(A, B)$, if and only if $\operatorname{det}\left(\begin{array}{ccc}x & y & z-R \\ v & -u & a \\ u & v & b\end{array}\right)=0$. Using (9) and (22), we get (23).

Combining Propositions 18, 8, and 20 we get the following result.

Corollary 21 (recognition of ruled surface offsets). Assume $\left(^{*}\right)$. If through each point of $\Phi$ there passes an oriented cylinder of fixed radius $R$ which is tangent to $\Phi$ along a continuous curve (containing the point), has inwards oriented normals and the axis nonparallel to the plane $z=0$, then for each $(x, y) \in D$ the equations (10), (22), (23) have a common real solution (u, v). 


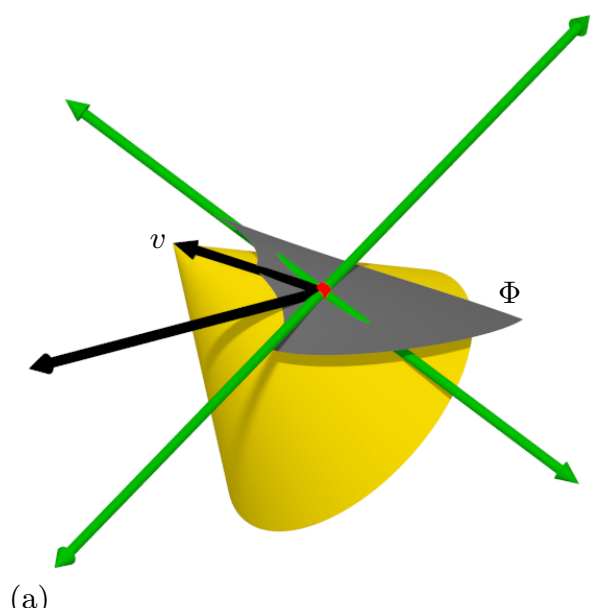

(a)

(b)

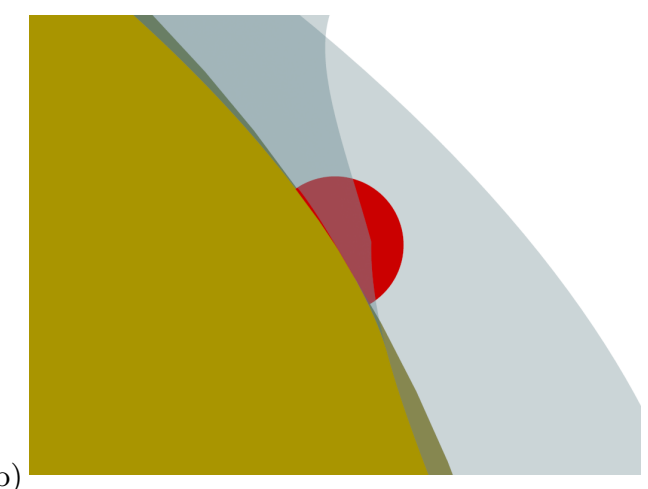

Figure 9: Reconstruction of an exact envelope. (a) An exact envelope $\Phi$ (dark) is generated from the function $f(x, y)=\frac{y^{2}}{x^{2}+y^{2}}$ by applying (19). The candidate tangent directions, i.e., the rulings, where third order contact with osculating cones having the opening angle $\theta=30^{\circ}$ occurs, are computed via (12), (19), and (21). The endpoints of the vectors correspond to the vertices of the hyperosculating cones. Our algorithm detects six positions with third order contact (green), including two rulings (black) that correspond to the exact generators. One generating cone (yellow) is shown. (b) A zoom-in view from the vertex $v$ of the cone. Observe the perfect local match between the cone and the surface (rendered in transparent) in a neighborhood of the contact point (red).

We keep just 3 equations in 2 variables $u$ and $v$ because it is already a nontrivial restriction on the function $f$. Geometrically the equations mean that there is a cylinder of radius $R$ having contact of order 3 with $\Phi$ (viewed as plane sets).

Problem 22. Is the reciprocal assertion in Corollary 21 true (if "each point" is replaced by "a generic point")? Find analogous characterizations of canal and pipe surfaces.

\section{Results and applications in CNC machining}

In this section, we show how the proposed analysis of third order contact can be used in the context of 5-axis flank CNC machining with conical tools. First, we test our algorithm on an exact envelope, showing that we reconstruct the generators of the envelope.

Example 23. Reconstruction of an exact generator. Take a particular surface that is an exact envelope of one-parameter family of cones. In the isotropic space, consider the graph of the function $f(x, y)=\frac{y^{2}}{x^{2}+y^{2}}$. The graph contains a family of isotropic circles whose top views are Euclidean circles passing through the origin $(0,0)$ and having radius $\frac{1}{2} \cot \theta$. They are the stereographic projections of circles of intrinsic radius $\frac{\pi}{2}-\theta$ passing through $(0,0,1)$ on the unit sphere. In the design space, they correspond to a motion of a cone with the opening angle $\theta=30^{\circ}$, see Fig. 9. We validated our approach by reconstructing the exact generator. Observe that there are two positions of the generating cone (as there are two isotropic circles passing through the point of the graph). One of the generating cones is shown in yellow in Fig. 9.

To further validate our approach, we tested to what extent one may have inexact data, and yet reconstruct the exact solution.

Example 24. Stability. We conducted a stability test as follows. We took the exact envelope generated from the function $f(x, y)=\frac{y^{2}}{x^{2}+y^{2}}$ by applying (19), perturbed the sampled tangent planes, and mapped these planes back to the isotropic space, see Fig. 10. The tangent plane perturbation was achieved by adding a random noise to the surface normals as follows. Let $\left\{\mathbf{d}_{1}, \mathbf{d}_{2}, \mathbf{n}\right\}$ be an orthonormal frame at a contact point, $\mathbf{n}$ being the unit normal. We define $\mathbf{v}=\alpha_{1} \mathbf{d}_{1}+\alpha_{2} \mathbf{d}_{2}$ and the perturbed normal as $\tilde{\mathbf{n}}=\frac{\mathbf{n}+\mathbf{v}}{\|\mathbf{n}+\mathbf{v}\|}$, where $\alpha_{1}=r \cos (\phi), \alpha_{2}=r \sin (\phi)$. The angle $\phi$ is randomly sampled from $[-\pi, \pi]$ and the random deviation is controlled via the parameter $r$ which is set to $r=0.1$ in the example shown in Fig. 10(a). The reconstruction of the isotropic circles from the exact and perturbed data are shown Fig. 10(b) and reconstruction of the hyperosculating cones is shown in Fig. 10(c).

Our last example is an industrial benchmark data set, namely a blade of an impeller. This blade is a general free-form surface and we show the hyperosculating positions of fixed cone attached to it.

Example 25. Industrial benchmark. The hyperosculating configurations, see Fig. 11, can be used for initialization of pathplanning algorithm of 5-axis flank CNC machining with conical milling tools [8]. Observe that only some hyperosculating cones can be used as candidates for the tool position due penetration of the cone with the neighboring blades. A sequence of positions at several hyperbolic contact points is shown in Fig. 11(h). However, the distance between the vertex of the cone and the contact 


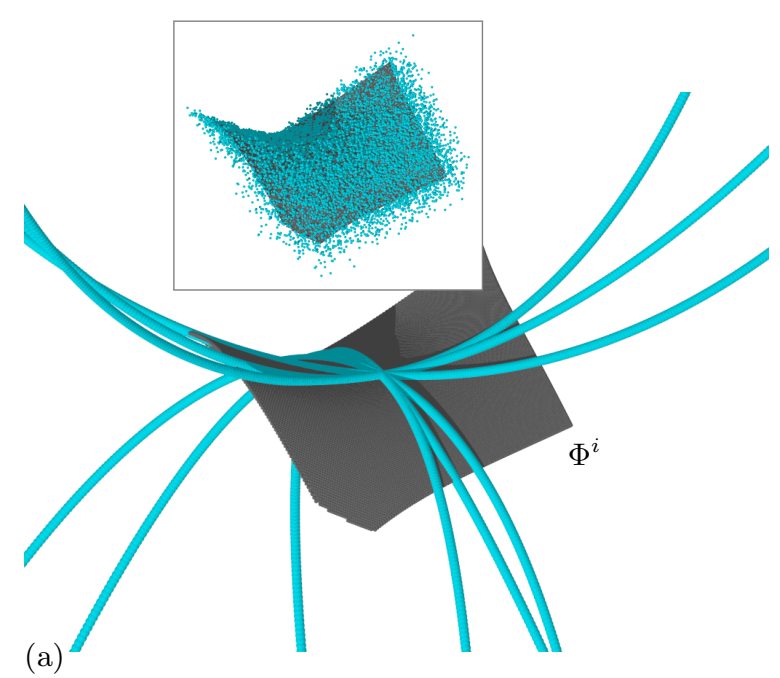

(a)

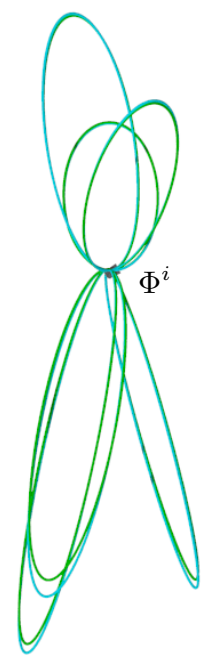

b)

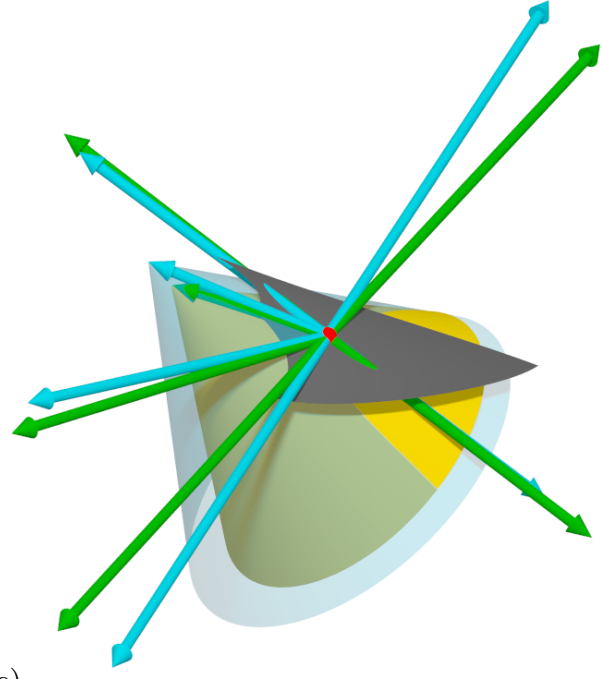

(c)

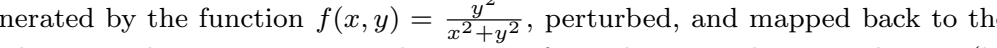

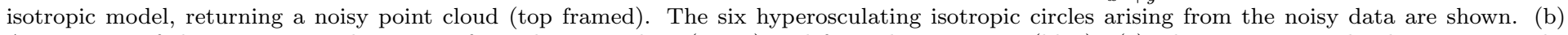

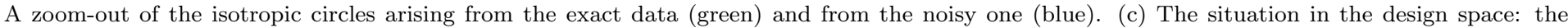

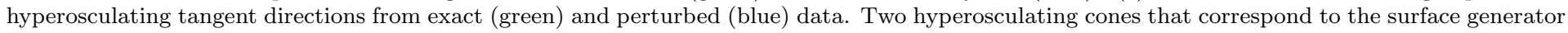
(yellow) and its approximation from noisy data (transparent) are shown.

point, i.e., the tool size, varies. Therefore, to design a path planning algorithm using these hyperosculating positions is a nontrivial problem as one needs to first search for an optimal tool size (contact point-vertex distance) that gives a sufficient number of tool positions in terms of surface coverage, and then design smooth milling paths through these positions. These issues as well as selection of a suitable opening angle go beyond this paper.

\section{Computational and implementation details}

To compute a higher-order contact cone at some $\mathbf{r} \in \Phi$, we use a set of neighboring points, denoted by $W$. These points can be samples and/or neighboring vertices of $\mathbf{r}$. Then a local coordinate system is defined as follows. The origin of the system is defined as the barycenter of $W$. The average normal of all data points in $W$ is set to be the $z$-axis, the $x$-axis is a random orthogonal vector, and the $y$-axis is the cross product of $z$ - and $x$ - axis vectors. After we have the local coordinate system, the coordinates of all points in $W$ are computed with respect to this local coordinate frame. Then the tangent planes to $\Phi$ at the points of $W$ are mapped to the isotropic model via (5) and we fit a biquintic polynomial to the image points by the least squares fitting method. This construction results in an approximation of $\Phi^{i}$ (more precisely, of the part arising from a neighborhood of $\mathbf{r}$ in $\Phi$ ) by the graph of a bivariate polynomial $f$. The polynomial $f$ is then used to compute the hyperosculationg positions of the cone, see Propositions 8, 16, 20.

The neighborhood $W$ is defined as follows. For the sake of the simplicity of the implementation, we take vertices within a ball of predefined radius (set to 0.2 in our implementation for surfaces with normalized bounding box). We compute the deviation of the surface normals at all points of $W$ with respect to the $z$-axis to guarantee that the patch is of Monge's type. We bisect the neighborhood diameter in the case when this condition is violated. This preprocessing aims at minimizing the distortion of mapping (5). Note that one can have vertices from other parts of the surface inside a Euclidean ball (for not well-behaved surfaces) and should prune these out, however, we have not encountered this issue for the test surfaces we used.

The whole computation of hyperosculating cones at a given point is real-time on a standard PC.

\section{Conclusion and future research}

We have derived necessary and sufficient conditions on a surface to be an envelope of a one-parameter family of congruent rotational cones. Such a surface can be milled by flank CNC machining with an appropriate conical tool in a single trace (provided that the motion is collision free and technical constraints on available tool sizes, machine workspace etc. are fulfilled as well). This characterization comes in form of nonlinear PDEs. On our way towards that, we discussed similar PDEs for ruled surfaces, and for completeness, we addressed envelopes of cylinders as well.

The derivation of our results uses contact order in the space of planes and the isotropic model of Laguerre geometry. It also led to conditions for higher order contact between rotational cones and a general surface, but contact is meant here in the space of planes. Contact in the standard point sense would not be useful anyway. We have shown (Section 4) that contact order in the space of planes is the right concept to get higher order contact between a surface generated by a conical (or cylindrical) tool and the target surface to be machined. 


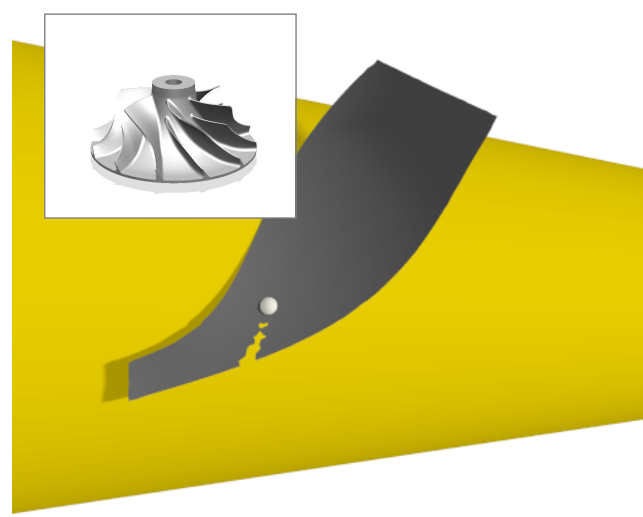

(a)

(d)

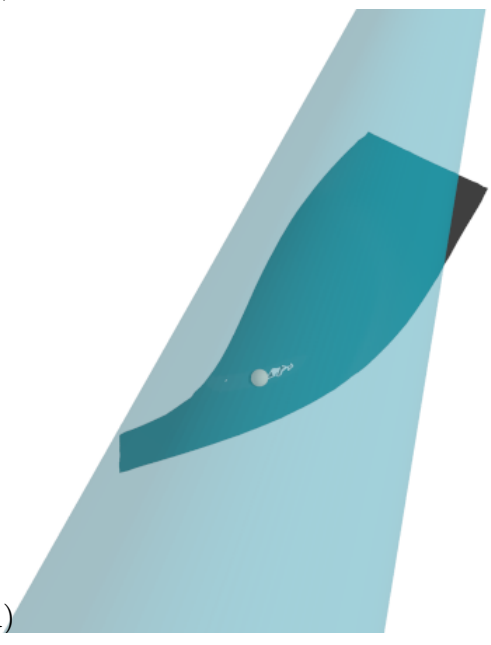

(b)

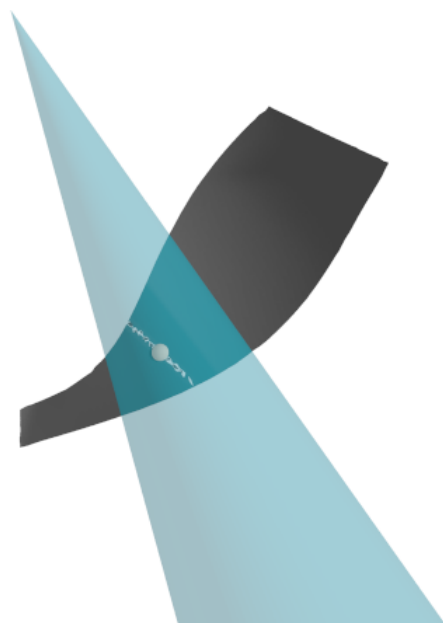

(c)

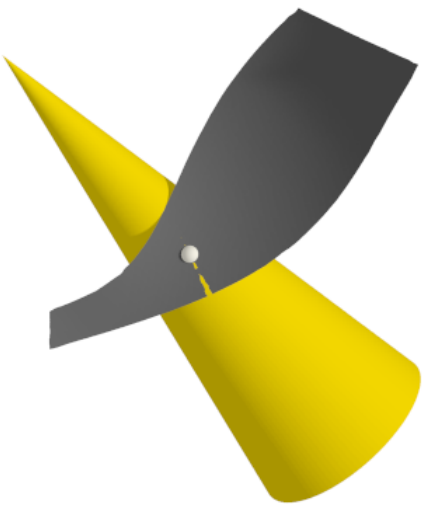

(e)

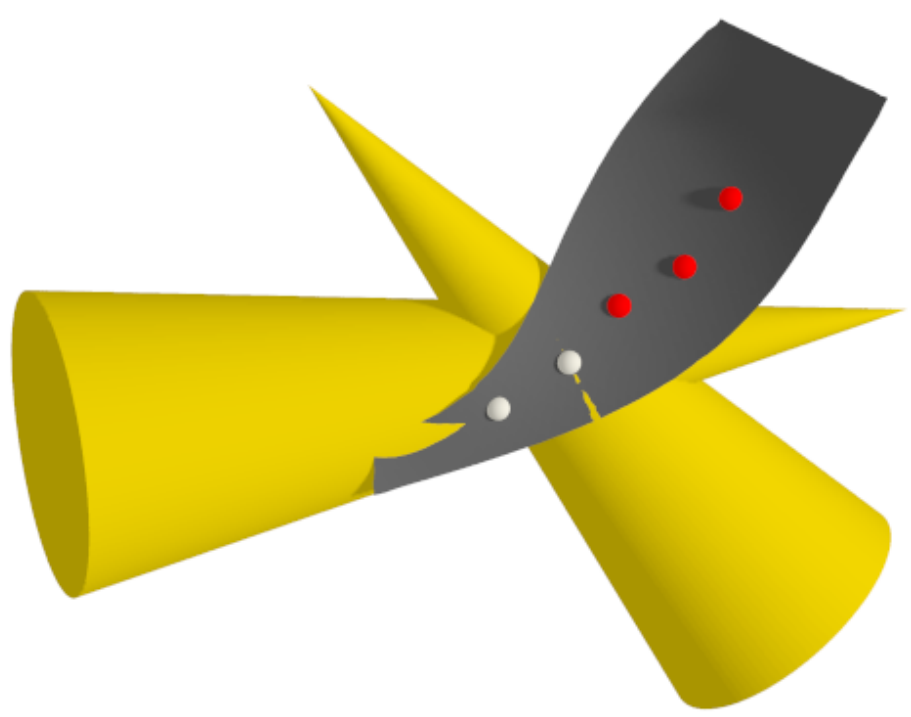

(f) (h)
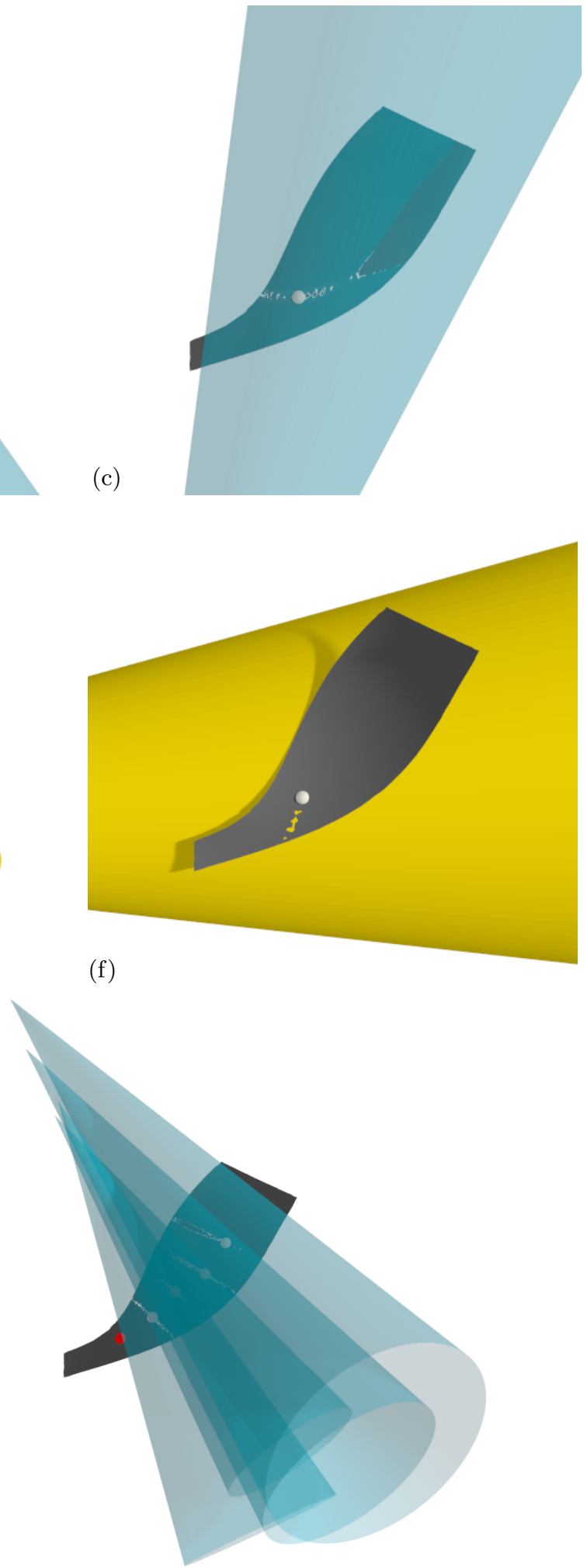

Figure 11: Industrial data. (a-f) Six positions of a cone with a specific opening angle $\theta=10^{\circ}$ that hyperosculates a blade of the impeller (framed) at contact point (white) are shown. The yellow cones approximate the blade "from-below", the transparent cones "from-above". Due to collision with other blades, only configurations (b) and (e) can be considered for flank CNC machining. (g) At five, user-selected, contact points, the hyperosculating cones are computed and suitable cones are shown. The red points indicate that there are no suitable hyperosculating cones from-below. (h) The sequence of hyperosculating cones from-above.

Finally, we provided some initial computational results which indicate that the concepts are useful for the development of algorithms for path planning in 5-axis flank CNC machining with conical tools. This is the part where future research could continue. The high order contact positions found according to our results should serve as appropriate initial tool positions for 
further optimization. A main research goal to be addressed is a complete coverage of a given design surface by well fitting envelopes of a moving tool, keeping the so-called scallop heights between neighboring machined strips as small as possible. Ideally, one could try to obtain scallop height free results in the sense that neighboring machined strips join smoothly. Even direct surface design could be guided by the fabrication with a certain technology, especially when very high accuracy is required. This would amount to the design of surfaces composed of surface strips which can be produced precisely with a certain technology.

\section{Appendix A. Reconstruction of rulings on ruled surfaces (Proof of Theorem 1)}

In this appendix we prove Theorem 1, and in the process give an "algorithm" to reconstruct the rulings on the graph of a function $f$ satisfying (2)-(3), and show how the technical assumptions in the theorem emerge naturally.

The plan of the argument is as follows. The implications $1 \Longrightarrow 2 \Longleftrightarrow 3$ have been essentially proved in Section 3 . To prove $3 \Longrightarrow 1$, first we consider separately the particular case of a developable surface. This case is classical, hence we just provide references. For a nondevelopable surface, we pick up a suitable normalized solution of system (1) at each point to get a smooth vector field (directions of ruling projections). Then we use the system to prove that the integral curves of the field are straight line segments and the restriction of $f$ to these segments is linear. Such reconstruction works for a generic point on the surface.

So, assume (2)-(3) and decompose the domain of the function $f$ into 3 subsets:

1. the interior of the set where $f_{x x} f_{y y}-f_{x y}^{2}=0$, i.e. the Gaussian curvature vanishes and the surface is developable;

2. the boundary of the set where $f_{x x} f_{y y}-f_{x y}^{2}=0$;

3. the interior of the set where $f_{x x} f_{y y}-f_{x y}^{2}<0$, i.e. the Gaussian curvature is negative.

\section{Developable surfaces}

Take a point in subset 1 . If $f_{x x}, f_{y y}, f_{x y}$ do not vanish simultaneously, then the line passing through the point and pointing in the asymptotic direction $\left(f_{y y},-f_{x y}, f_{x} f_{y y}-f_{y} f_{x y}\right)$ is the required ruling [12, Proposition 1 in $\left.\S 5.8\right]$. On the boundary of the subset where $f_{x x}, f_{y y}, f_{x y}$ do not vanish simultaneously, the required ruling points in the limiting direction of the asymptotic directions at nearby points [12, Proposition 3 in $\S 5.8]$. In the interior of the subset where $f_{x x}=f_{y y}=f_{x y}=0$, the graph is locally planar, hence there are infinitely many rulings [12, Proposition 5 in $§ 3.2]$.

\section{Technical conventions}

Now consider subset 2. Theoretically, it can be a complicated fractal (possibly of nonzero Lebesque measure; see also [12, Example 1 in §5.8]). Practically the boundary (if nonempty at all) is a curve on the surface, hence "negligible" (although still sensible because our algorithm may become unstable near it). To avoid too much technicalities while keeping our work mathematically correct, we prefer just to drop subset 2 and limit ourselves to "generic" points on a surface; see condition 1 in Theorem 1.

Definition. A negligible subset is a countable union of subsets such that the closure of each one has no interior points. We say that an assertion holds at a generic point, if it holds outside a negligible set.

For instance, the boundary of the zero set of a continuous function is always negligible. On the other hand, whatever small disc in the plane is not negligible (this is the Baire category theorem).

Notice that the derivation of (2)-(3) in Section 3 remains true, even if we assume that the surface contains a line segment through a generic point rather than through each point; see condition 1 in Theorem 1 . Indeed, then (2)-(3) hold at a generic point $(x, y)$; but since their left-hand sides are continuous, they must hold everywhere. Also, in Section 3 we have already proved that (2)-(3) is equivalent to the existence of a nonzero real solution of (1).

\section{Yet another technical issue}

Now consider subset 3. Assume that (1) has a nonzero real solution $(u, v)$ at each point $(x, y)$. We would like to pick up a nonzero solution $(u(x, y), v(x, y))$ smoothly $\left(C^{1}\right)$ depending on the point $(x, y)$. This is not possible in general: for instance, the solutions of the system

$$
\left\{\begin{array}{l}
u^{2}-v^{2}=0, \\
x u^{3}-|x| v^{3}=0 ;
\end{array}\right.
$$

are proportional to $(1,1)$ for $x \geq 0$ and to $(1,-1)$ for $x \leq 0$. Thus we have to restrict to a smaller domain as follows.

Consider the auxiliary system consisting of the first equation of (1) and the equation $u^{2}+v^{2}=1$. The former is quadratic with the discriminant $f_{x x} f_{y y}-f_{x y}{ }^{2}<0$, hence defines a pair of lines passing through the origin in the $(u, v)$ plane. The latter equation defines a circle transversal to the lines. Hence the system has exactly 4 solutions, none of which are multiple. By the implicit function theorem, in a sufficiently small neighborhood of any point $\left(x_{0}, y_{0}\right)$ the solutions form 4 smooth branches $\left(u_{k}(x, y), v_{k}(x, y)\right)$, where $k=1,2,3,4$.

For each $k=1,2,3,4$ consider the closed subset where $\left(u_{k}(x, y), v_{k}(x, y)\right)$ satisfies the second equation of $(1)$ as well. These 4 subsets cover the whole neighborhood in question and have negligible boundary. Thus a generic point belongs to the interior of one of these subsets. 
This is equivalent to (recall notation (15))

$$
(v+\dot{u}) \underbrace{\left(x\left(x^{2}+y^{2}+1+2 x u+2 y v\right)-4 u \tan ^{2} \theta\right)}_{\tilde{u}}=(u-\dot{v}) \underbrace{\left(y\left(x^{2}+y^{2}+1+2 x u+2 y v\right)-4 v \tan ^{2} \theta\right)}_{\tilde{v}} .
$$

Here $(\tilde{u}, \tilde{v})$ is half of the gradient of the left-hand side of $(8)$ considered as a function in $(u, v)$. Thus $(\tilde{u}, \tilde{v}) \neq(0,0)$ because otherwise $(u, v)$ would be a multiple solution of the system of equations (8) and (10). This implies that there is a function $g=g(t)($ e.g., $g=(\dot{v}-u) / \tilde{u}$ for $\tilde{u} \neq 0)$ such that

$$
\left\{\begin{array}{l}
\dot{u}=-v-g \tilde{v} \\
\dot{v}=u+g \tilde{u}
\end{array}\right.
$$

568 Here we have essentially relied on a particular form of constraint (8).

Let us now switch to (10). Differentiating with respect to $t$, substituting (B.1) and subtracting (11), we get

$$
\begin{aligned}
f_{x x x x} v^{4}- & 4 f_{x x x y} v^{3} u+6 f_{x x y y} v^{2} u^{2}-4 f_{x y y y} v u^{3}+f_{y y y y} u^{4}+3 u v^{2} f_{x x x}+3 v\left(v^{2}-2 u^{2}\right) f_{x x y}+3 u\left(u^{2}-2 v^{2}\right) f_{x y y}+3 u^{2} v f_{y y y} \\
& +3 v^{2} \dot{v} f_{x x x}-3 v(2 u \dot{v}+v \dot{u}) f_{x x y}+3 u(2 v \dot{u}+u \dot{v}) f_{x y y}-3 u^{2} \dot{u} f_{y y y}+3(u \dot{v}+v \dot{u})\left(f_{x x}-f_{y y}\right)+6(v \dot{v}-u \dot{u}) f_{x y} \\
= & 3 g\left(f_{x x x} v^{2} \tilde{u}+f_{x x y} v(v \tilde{v}-2 u \tilde{u})+f_{x y y} u(u \tilde{u}-2 v \tilde{v})+f_{y y y} u^{2} \tilde{v}+\left(f_{x x}-f_{y y}\right)(u \tilde{u}-v \tilde{v})+2 f_{x y}(u \tilde{v}+v \tilde{u})\right)=g J=0
\end{aligned}
$$


where $J$ is the Jacobian of the system of equations (8) and (10) in $u$ and $v$. Here $J \neq 0$ because $(u, v)$ is not a multiple solution; thus $g(t)=0$ identically.

(The expression $g J$ in the right-hand side is what one should actually expect: The left-hand side is obviously linear in $g$ and vanishes for $g=0$ because (11) was obtained from (10) by differentiating along a circle. The coefficient before $g$ is $J$ because $(\tilde{u}, \tilde{v})$ is half of the gradient of the left-hand side of (8).)

Then by $($ B.1) we get $(\dot{u}, \dot{v})=(-v, u)$. Here $(-v, u) \neq 0$ by (8). Thus the integral curve $(x(t), y(t))$ is a circular arc parametrized by the first two equations of (7) with $x=x(0), y=y(0), u=u(x(0), y(0)), v=v(x(0), y(0))$.

In particular, this means that the integral curve is contained in the top view of the conic $C_{x(0), y(0)}$. By a similar argument, the same curve is contained in the top view of each conic $C_{x(t), y(t)}$ (which a priori could be different from $C_{x(0), y(0)}$ ).

Clearly, for each fixed $a, b, z$ and each $t$ the function $z(t)$ given by the third equation of (7) satisfies

$$
\frac{d^{3} z(t)}{d t^{3}}+\frac{d z(t)}{d t}=0
$$

Since $C_{x(t), y(t)}$ has contact of order 3 with the graph of $f$, it follows that for each $t$ sufficiently close to 0 also

$$
\frac{d^{3} f(x(t), y(t))}{d t^{3}}+\frac{d f(x(t), y(t))}{d t}=0 .
$$

Thus $f(x(t), y(t))=z^{\prime}+a^{\prime} \sin t+b^{\prime}(1-\cos t)$ for some $z^{\prime}, a^{\prime}, b^{\prime} \in \mathbb{R}$. Since the graph of $f$ has contact of order 2 with $C_{x(0), y(0)}$, it follows that $z^{\prime}=z(x(0), y(0)), a^{\prime}=a(x(0), y(0)), b^{\prime}=b(x(0), y(0))$, and the graph must contain an arc of the conic $C_{x(0), y(0)}$ completely. This completes the proof of Theorem 10.

\section{Acknowledgements}

Three of the four coauthors are grateful to King Abdullah University of Science and Technology, where they met altogether and started this project. The authors are also grateful to R. Bryant, S. Ivanov, and A. Skopenkov for useful discussions. The first author has been supported within the framework of the Academic Fund Program at the National Research University Higher School of Economics (HSE) in 2018-2019 (grant N18-01-0023) and by the Russian Academic Excellence Project "5-100". The second author has been partially supported by the National Natural Science Foundation of China $(62072139,61672187)$. The third author has been partially supported by Spanish Ministry of Science, Innovation and Universities: Ramón y Cajal with reference RYC-2017-22649 and the European Union's Horizon 2020 research and innovation programme under agreement No. 862025 .

\section{References}

[1] M. Bartoň, L. Shi, H. Pottmann, M. Kilian, and J. Wallner, Circular arc snakes and kinematic surface generation, Computer Graphics Forum 32 (2013), no. 1, 1-10.

[2] Wilhelm Blaschke, Vorlesungen über differentialgeometrie, vol. 2, Springer, 1923.

[3] _ Vorlesungen über differentialgeometrie, vol. 3, Springer, 1929.

[4] Pengbo Bo and Michael Bartoň, On initialization of milling paths for flank CNC machining of free-form surfaces with curvature-varying milling tools, Computer Aided Geometric Design 71 (2019), 30-42.

[5] Pengbo Bo, Michael Barton̆, and Helmut Pottmann, Automatic fitting of conical envelopes to free-form surfaces for flank CNC machining, Computer-Aided Design 91 (2017), 84-94.

[6] Pengbo Bo, Helmut Pottmann, Martin Kilian, Wenping Wang, and Johannes Wallner, Circular arc structures, vol. 30, ACM, 2011.

[7] R. Bryant, Partial differential equation for ruled surfaces, (2011), https://mathoverflow.net/q/77967.

[8] Amaia Calleja, Pengbo Bo, Haizea González, Michael Bartoň, and Luis Norberto López de Lacalle, Highly accurate 5-axis flank CNC machining with conical tools, The International Journal of Advanced Manufacturing Technology (2018), 1-11.

[9] T. Cecil, Lie sphere geometry, Springer, 1992.

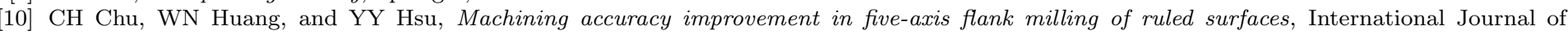
Machine Tools and Manufacture 48 (2008), no. 7, 914-921.

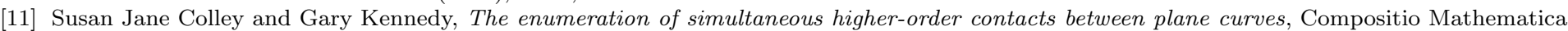
93 (1994), no. 2, 171-209.

[12] Manfredo do Carmo, Differential geometry of curves and surfaces, Prentice-Hall, 1976.

[13] Marc Dohm and Severinas Zube, The implicit equation of a canal surface, Journal of Symbolic Computation 44 (2009), no. 2, 111 - 130.

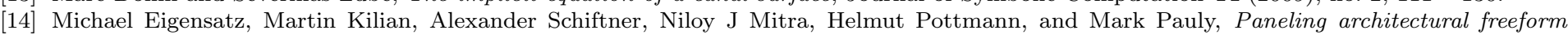
surfaces, ACM transactions on graphics (TOG), vol. 29, ACM, 2010, p. 45.

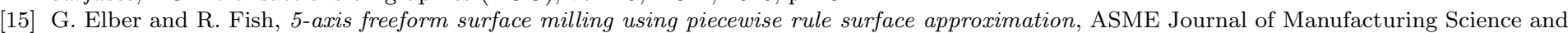
Engineering 119 (1997), no. 3, 383-387.

[16] Ben Ezair and Gershon Elber, Automatic generation of globally assured collision free orientations for 5-axis ball-end tool-paths, Computer-Aided Design 102 (2018), 171-181.

[17] Simon Flöry, Yukie Nagai, Florin Isvoranu, Helmut Pottmann, and Johannes Wallner, Ruled free forms, Advances in Architectural Geometry (Lars Hesselgren, Shrikant Sharma, Johannes Wallner, Niccolo Baldassini, Philippe Bompas, and Jacques Raynaud, eds.), Springer, 2012, pp. $57-66$.

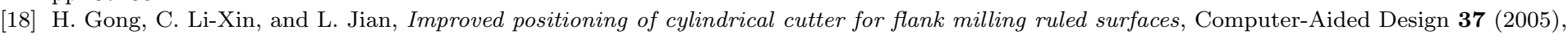
$1205-1213$ 
19] H. Gong and N. Wang, Optimize tool paths of flank milling with generic cutters based on approximation using the tool envelope surface, Computer-Aided Design 41 (2009), no. 12, 981-989.

[20] Josef Hoschek and Ulrich Schwanecke, Interpolation and approximation with ruled surfaces, The mathematics of surfaces 8 (1998), $213-231$.

[21] R. Krasauskas and C. Mäurer, Studying cyclides with Laguerre geometry, Computer Aided Geometric Design 17 (2000), no. 2 , 101 - 126.

[22] Rimvydas Krasauskas and Severinas Zube, Canal surfaces defined by quadratic families of spheres, pp. 79-92, 01 2008.

[23] Erwin Kruppa, Analytische und konstruktive Differentialgeometrie, Springer, 1957.

[24] C. Li, S. Bedi, and S. Mann, Flank milling of a ruled surface with conical tools - an optimization approach, Int. J. Adv. Manuf. Technol. 29 (2006), 1115i-1124.

[25] Flank millable surface design with conical and barrel tools, Computer- Aided Design and Applications 5 (2008), 461-470.

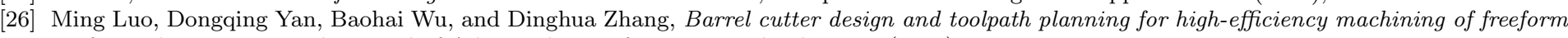
surface, The International Journal of Advanced Manufacturing Technology 85 (2016), no. 9-12, 2495-2503.

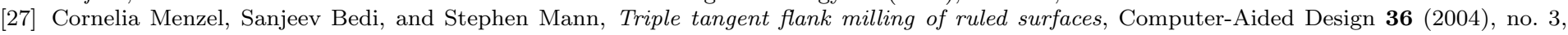
$289-296$.

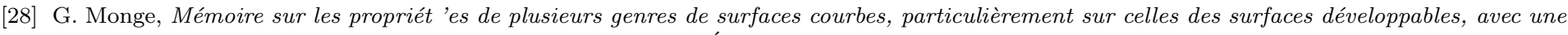
application à la théorie des ombres et des pénombres, Savans Étrangers 9 (1780), 382-440.

[29] James A Montaldi et al., Surfaces in 3-space and their contact with circles, Journal of Differential Geometry 23 (1986), no. 2, $109-126$.

[30] M. Peternell and H. Pottmann, A Laguerre geometric approach to rational offsets, Comp. Aid. Geom. Des. 15 (1998), $223-249$.

[31] H. Pottmann and M. Peternell, Applications of Laguerre geometry in CAGD, Comp. Aid. Geom. Des. 15 (1998), $165-186$.

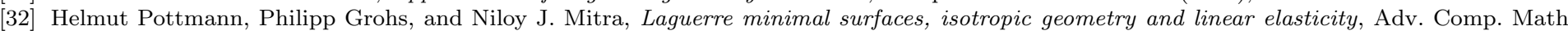
31 (2009), 391-419.

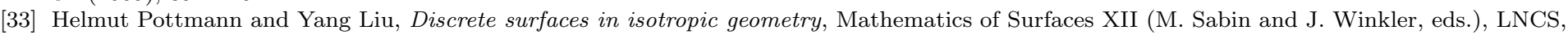
vol. 4647, Springer, 2007, pp. 431-363.

[34] Helmut Pottmann and Johannes Wallner, Computational line geometry, Springer, 2001.

[35] Michael Rabinovich, Tim Hoffmann, and Olga Sorkine-Hornung, Discrete geodesic nets for modeling developable surfaces, ACM Transactions on Graphics (ToG) 37 (2018), no. 2, 16.

[36] J. Redonnet, W. Rubio, and G. Dessein, Side milling of ruled surfaces; optimum positioning of the milling cutter and calculation of interference, The International Journal of Advanced Manufacturing Technology 14 (1998), no. 7, 459-465.

[37] Hans Sachs, Isotrope Geometrie des Raumes, Vieweg, 1990.

38] M. Skopenkov, H. Pottmann, and P. Grohs, Ruled laguerre minimal surfaces, Math. Z. 272 (2012), no. 1-2, 645-674.

[39] Justin Solomon, Etienne Vouga, Max Wardetzky, and Eitan Grinspun, Flexible developable surfaces, Computer Graphics Forum, vol. 31, Wiley Online Library, 2012, pp. 1567-1576.

[40] K. Sprott and B. Ravani, Cylindrical milling of ruled surfaces, Int. J. Adv. Manuf. Technol. 38 (2008), 649-656.

[41] Jacob Subag and Gershon Elber, Piecewise developable surface approximation of general nurbs surfaces, with global error bounds, International Conference on Geometric Modeling and Processing, Springer, 2006, pp. 143-156.

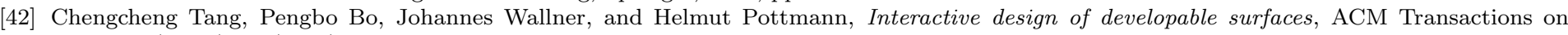
Graphics (TOG) 35 (2016), no. 2, 12.

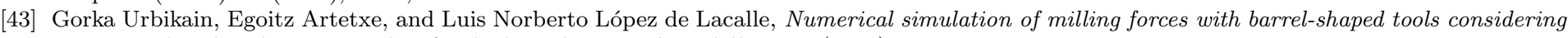
runout and tool inclination angles, Applied Mathematical Modelling 47 (2017), 619-636.

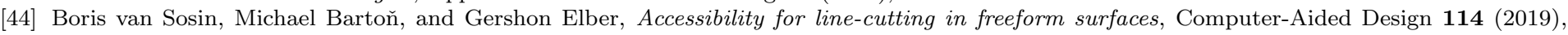
$202-214$.

[45] C.C.L. Wang and G. Elber, Multi-dimensional dynamic programming in ruled surface fitting, Computer-Aided Design 51 (2014), 39-49.

[46] X. C. Wang, S. K. Ghosh, Y.B. Li, and X. T. Wu, Curvature catering - a new approach in manufacture of sculptured surfaces (part 1. theorem), Journal of Materials Processing Technology 38 (1993), no. 1-2, 159-175.

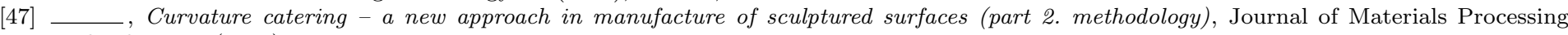
Technology 38 (1993), no. 1-2, 177-193.

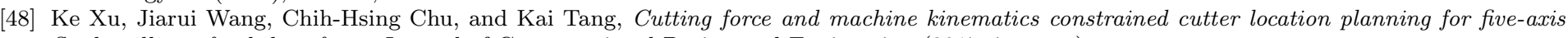
flank milling of ruled surfaces, Journal of Computational Design and Engineering (2017, in press).

[49] Xiuzi Ye and Takashi Maekawa, Differential geometry of intersection curves of two surfaces, Computer Aided Geometric Design 16 (1999), no. 8, 767-788.

[50] Kim Y.J., Bartoň M., Elber G., and Pottmann H., Precise gouging-free tool orientations for 5-axis CNC machining, Computer-Aided Design 58 (2015), 220-229.

51] Liang Yu, Lei Zhong, and Yuhan Wang, Optimizing tool size and tool path of five-axis flank milling with bounded constraints via normal mapping, Advances in Mechanical Engineering 9 (2017), no. 10, 1687814017724704.

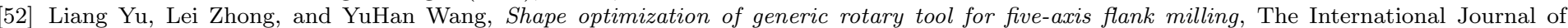
Advanced Manufacturing Technology 93 (2017), no. 5-8, 2921-2931.

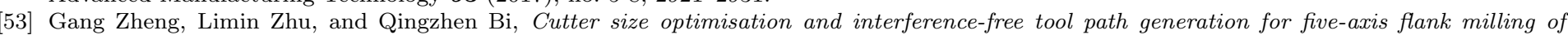
centrifugal impellers, International Journal of Production Research 50 (2012), no. 23, 6667-6678.

[54] L. Zhu, H. Ding, and Y. Xiong, Simultaneous optimization of tool path and shape for five-axis flank milling, Comput. Aided Des. 44 (2012), 1229-1234.

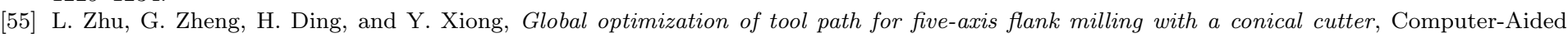
Design 42 (2010), no. 10, 903-910. 